\title{
An Integrated Traverse Planner and Analysis Tool for Planetary Exploration
}

\author{
Aaron W. Johnson*, Jeffrey A. Hoffman ${ }^{\dagger}$, and Dava J. Newman \\ Massachusetts Institute of Technology, Cambridge, MA, 02139, United States \\ Erwan M. Mazarico ${ }^{\S, * *}$ \\ Massachusetts Institute of Technology, Cambridge, MA, 02139, United States \\ NASA Goddard Space Flight Center, Greenbelt, MD, 20771, United States \\ and \\ Maria T. Zuber ${ }^{\dagger}$ \\ Massachusetts Institute of Technology, Cambridge, MA, United States.
}

\begin{abstract}
Future planetary explorations will require surface traverses of unprecedented frequency, length, and duration. As a result, there is need for exploration support tools to maximize productivity, scientific return, and safety. The Massachusetts Institute of Technology is currently developing such a system, called the Surface Exploration Traverse Analysis and Navigation Tool (SEXTANT). The goal of this system is twofold: to allow for realistic simulations of traverses in order to assist with hardware design, and to give astronauts an aid that will allow for more autonomy in traverse planning and re-planning. SEXTANT is a MATLAB-based tool that incorporates a lunar elevation model created from data from the Lunar Orbiter Laser Altimeter instrument aboard the Lunar Reconnaissance Orbiter spacecraft. To assist in traverse planning, SEXTANT determines the most efficient path across a planetary surface for astronauts or transportation rovers between user-specified Activity Points. The path efficiency is derived from any number of metrics: the traverse distance, traverse time, or the explorer's energy consumption. The generated path, display of traverse obstacles, and selection of Activity Points are visualized in a 3D mapping interface. After a traverse has been planned, SEXTANT is capable of computing the most efficient path back home, or "walkback", from any point along the traverse - an important capability for emergency operations. SEXTANT also has the ability to determine shadowed and sunlit areas along a lunar traverse. This data is used to compute the thermal load on suited astronauts and the solar power generation capacity of rovers over the entire traverse. These both relate directly to the explorer's consumables, which place strict constraints on the traverse. This paper concludes by presenting three example traverses, detailing how SEXTANT can be used to plan and modify paths for both explorer types.
\end{abstract}

\section{Nomenclature}

$\alpha=$ terrain slope

$\alpha_{\text {suit }} \quad=$ absorptivity of the space suit

$A_{\perp} \quad=\quad$ space suit surface area perpendicular to the sun

\footnotetext{
${ }^{*}$ Research Assistant, Dept. of Aeronautics and Astronautics, Room 37-219, 77 Massachusetts Ave., Member, AIAA

$\dagger$ Professor of the Practice, Dept. of Aeronautics and Astronautics, Room 37-227, 77 Massachusetts Ave., Senior Member, AIAA

* Professor, Dept. of Aeronautics and Astronautics, Room 33-307, 77 Massachusetts Ave., Associate Fellow, AIAA

${ }^{\S}$ Research Scientist, Dept. of Earth, Atmospheric, and Planetary Sciences, Room 54-518, 77 Massachusetts Ave.

${ }^{* *}$ Research Scientist, Sciences and Exploration Directorate, Mail Stop 698

${ }^{\dagger}$ Professor, Dept. of Earth, Atmospheric, and Planetary Sciences, Room 54-518, 77 Massachusetts Ave.
}

1

American Institute of Aeronautics and Astronautics 


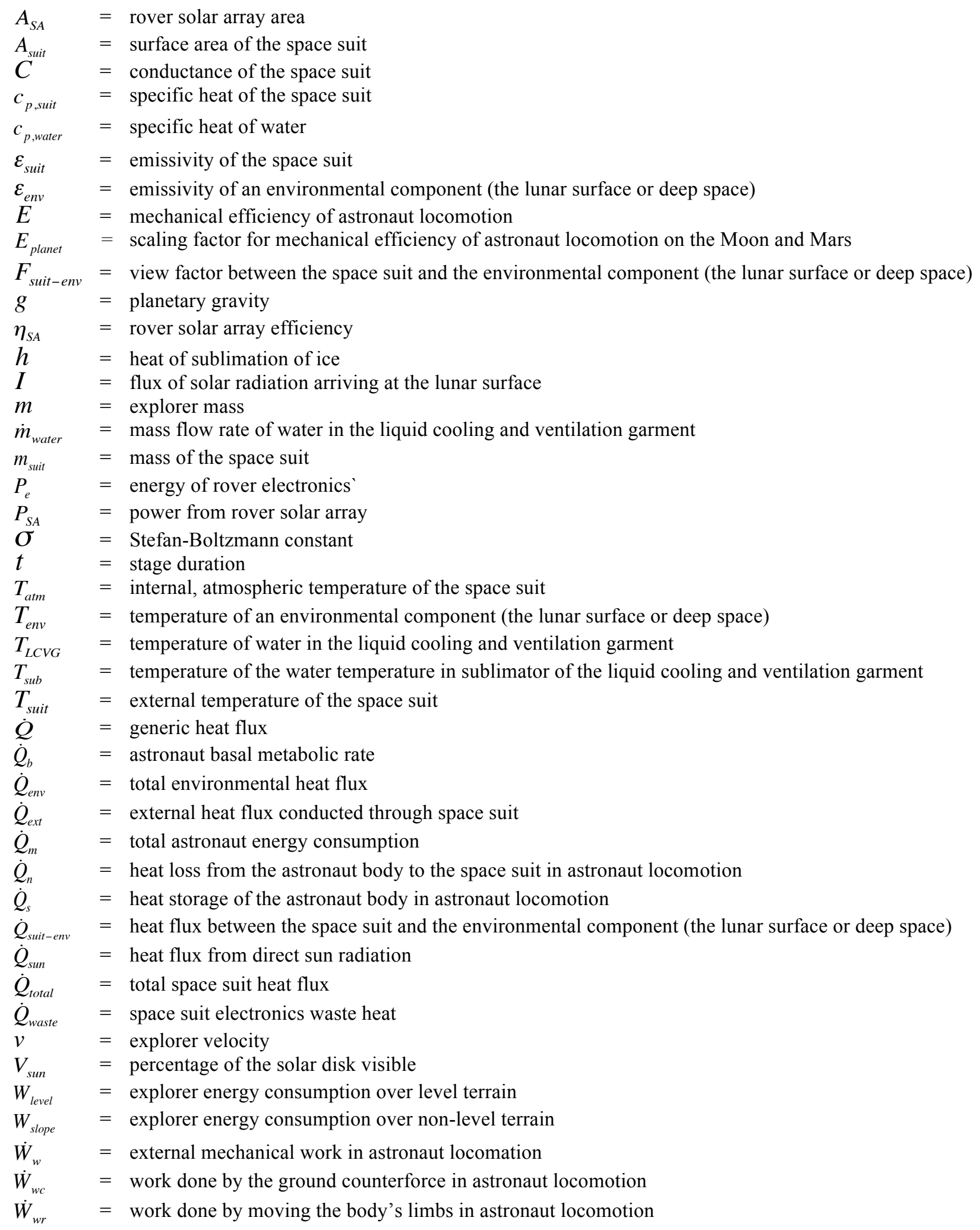

\section{Introduction}

GUTURE explorations of the Moon and Mars will require traverses of a scope and scale greater than any ever 1 attempted. To this point in time, there have only been six manned missions to the lunar surface, all during the 
United States' Apollo Program. The astronauts on these missions accomplished a total of 14 separate extravehicular activities (EVAs), the longest of which covered $20.4 \mathrm{~km}$ in 7 hours and 37 minutes during the Apollo 17 mission. During future explorations, traverses on foot and in robotic transportation rovers will be more frequent and much longer in time and distance. For example, under the most recent NASA lunar architecture, lunar space suits were being designed for 90 individual traverses on foot, each of up to 8 hours. ${ }^{1}$ This requirement is similar in duration to the longest Apollo EVA, as well as current Space Shuttle and ISS EVAs. However, a two-person pressurized vehicle called the Lunar Electric Rover was also being planned with the capability for 3-day traverses of up to $100 \mathrm{~km}$, or 14-day traverses of up to $1000 \mathrm{~km} .{ }^{1}$ Even the shorter end of this range is an order of magnitude farther and longer than the longest Apollo traverse. Martian traverses will employ similar hardware, and their limits will also be much greater than those during the Apollo program.

During the Apollo program, astronauts on EVA were confounded by specific challenges to navigation in the lunar environment. Unless compensated for, these and similar problems for the Martian surface, will be exacerbated by the increased frequency, length, and duration of future traverses. The second EVA of the Apollo 14 mission clearly demonstrates these difficulties. ${ }^{2}$ This EVA was the final walking EVA of the Apollo program, as all subsequent Apollo missions carried the Lunar Roving Vehicle (LRV). Commander Alan Shepard and Lunar Module Pilot Ed Mitchell left the Lunar Module with the goal of traveling to Cone Crater, approximately $1.5 \mathrm{~km}$ away. Shepard and Mitchell navigated by use of a paper map developed from photographs of the lunar surface, with craters identified as navigational landmarks. During their EVA, Shepard and Mitchell had difficulty in identifying specific craters by sight. This led to poor situational awareness, or confusion as to their exact position. Additionally, they had trouble judging distances and travel times on the lunar surface due to the lack of atmosphere and distortions from the helmet visor. ${ }^{3}$ As the EVA progressed, Shepard and Mitchell traveled up steep slopes, necessitating high amounts of exertion and more frequent rest stops. They continued to search for the rim of Cone Crater, knowing they were close but not being able to see it. Eventually, the astronauts used up their 30-minute EVA extension time and had to continue on with the EVA despite never reaching Cone Crater. Post-mission analysis indicated that they were only approximately 20 meters from the rim, but did not know it at the time. Similar difficulties will likely be encountered by astronauts exploring the Martian surface, with additional challenges posed by the harsh environment and climate.

Difficulties like these must be overcome if future surface explorations are going to be a success. To help mitigate these challenges and improve the ability to carry out explorations on planetary surfaces, the Massachusetts Institute of Technology (MIT) Man Vehicle Laboratory has developed a traverse planning tool called the Surface Exploration Traverse Analysis and Navigation Tool (SEXTANT). SEXTANT is a decision support aid designed to help users plan and optimize paths over a planetary surface for both suited astronauts on foot and transportation rovers. Its aim is to help analyze and present information to assist the user, rather than making and carrying out its own decision. When using SEXTANT, the user must process the information and assess the path's validity and choose whether or not it should be carried out. SEXTANT contains an interface with a 3D terrain elevation map over which the user can place points of interest, called Activity Points. Terrain obstacles are areas where the explorer cannot travel due to safety constraints, and are specified by a user-inputted maximum slope. After the user has specified the Activity Points, SEXTANT determines the most-efficient path between them based on the metric of traverse distance, time, or explorer energy consumption. SEXTANT also has the ability to determine the shadowing along a planned lunar traverse. This is then used to compute the thermal loading on an astronaut, and the power usage and generation for a transportation rover. These factors directly influence the consumables required to complete the traverse, which are the heater power and amount of water needed to replace ice sublimated away for cooling for the astronaut, and the battery energy for the rover.

\section{A. Decision Support Aids}

\section{Background}

One of the most important factors in designing a decision support aid like SEXTANT is the amount of automation that it should employ. Parasuraman et al. define automation as "the full or partial replacement of a function previously carried out by the human operator." ${ }^{4}$ The use of automation can help to increase the efficiency of carrying out a complex task and greatly decrease the amount of time required. However, too much or incorrect automation can lead to sub-optimal results in unanticipated situations, user complacency (called automation bias), and a loss of user situational awareness. ${ }^{5}$ Therefore, it is important to carefully choose the amount of automation in any decision support aid.

A great deal of research has investigated the effects of varying levels of automation in a decision support aid. Layton, Smith, and McCoy investigated an experimental decision support aid for re-planning commercial airplane flights around thunderstorms while en-route. ${ }^{6,7}$ The authors showed that automation can greatly help the user to

3

American Institute of Aeronautics and Astronautics 
arrive at "optimal" solutions when there is a large solution space. However, they also demonstrated that automation can be "brittle", meaning that it can fail and provide sub-optimal solutions in unanticipated situations. This can occur when the system's model of the "world" is inadequate or when it fails to consider relevant factors. To prevent brittleness, SEXTANT attempts to model the "world" (a lunar or planetary traverse) as accurately as possible. Brittleness is also compensated for by keeping the human user "in the loop", or involved with the task, as Layton, Smith, and McCoy suggest. SEXTANT accomplishes this by makingthe user specify the Activity Points. This gives the user control over the destinations of the traverse, and only allows the automation to plan the route between these specified locations.

Decision support aids for planning have been extensively used during the Mars Exploration Rover (MER) Spirit and Opportunity missions. ${ }^{8,9}$ The tools used during these missions are somewhat different from SEXTANT in that they are used for temporal scheduling, and not path planning. However, the lessons learned regarding automation are still very pertinent. During the MER missions, McCurdy observed that the automated planning tools were not used very frequently. ${ }^{9}$ McCurdy hypothesized that this was for two reasons: that it was impossible to capture all science constraints in the planning tools, and that the automatically-generated schedules were difficult to understand. In the next generation of Martian planning tools, the Phoenix Science Interface (PSI), changes were made to involve humans more in the decision process. PSI used passive constraint checking, where a plan was automatically generated and compared to constraints. If a violation was found, only suggestions for resolution were presented. It was the user's responsibility to ensure that the plan is viable. This is the same technique used by SEXTANT. Traverse paths are not optimized around any certain constraints. Rather, the traverse information is presented with respect to user-specified constraints, which shows how much margin there is, if any. The user can then make the decision on how to best modify the traverse to fit within the constraints.

\section{B. Traverse Planners for Planetary Surface Exploration}

Planning EVAs for each Apollo lunar mission began with establishing locations of scientific interest. As Bill Muehlberger, chief geologist for the Apollo 16 and 17 missions, stated, "The name of the game in traverse planning is maximum scientific return." 10 After sites of interest were selected and prioritized, traverses were planned by hand to maximize the return while remaining within all of the given constraints. The only path planning tools used were photomosaics and topographic maps produced by previous manned and unmanned missions. As a result, there was a great deal of uncertainty about how quickly and well the astronauts would be able to travel over the terrain. The planning team had to make assumptions that were not always correct, which caused problems during some of the EVAs when the astronauts could not travel as easily as expected. Furthermore, when these challenges were encountered, the astronauts did not have the ability to re-plan their traverses by themselves. They had to radio down to the engineers and scientists on Earth, who would re-plan the EVA and direct the astronauts from the ground.

Since the Apollo program, only a few researchers have looked at improving traverse planning tools. Wilkinson developed the Traverse Generation Assistant (TGA) for long-duration pressurized rovers traverses on Mars. ${ }^{11}$ While TGA is similar to SEXTANT in many ways, its model of the traverse is more limited than that of SEXTANT in a number of ways. Most significantly, TGA does not consider the rover's energy consumption during a traverse. Wilkinson does note that the metabolic cost of a traverse has been left out of the model because TGA is for planning rover traverses. This is true, but the rover energy cost for a traverse - analogous to an astronaut's metabolic expenditure - is a critical metric for determining whether or not the traverse is valid. A traverse often has distance or time constraints, but these are mainly designed to ensure that the explorer has enough consumables. For a rover, battery power is one of the most important consumables. SEXTANT allows the user to optimize a rover traverse on its energy cost, and presents the energy consumption for all planned traverses. This allows the user to clearly see if there will be enough consumables to complete the EVA.

Wood and Wood developed a traverse planning tool of sorts that does take into account the metabolic cost of travel; however, the goal of their tool is quite different than that of TGA or SEXTANT. ${ }^{12}$ Their tool is for use in archaeology on Earth, and is designed to compute geographic closeness on the metric of expended energy. Wood and Wood's planning tool is similar to SEXTANT in that both have the ability to optimize traverses on the metric of metabolic cost. In fact, both tools use the same metabolic cost equations developed by Santee et al. ${ }^{13}$ Even so, the capabilities of Wood and Wood's tool are less flexible than those SEXTANT. In Wood and Wood's tool, the user can only optimize the path between two points. In SEXTANT, the user can create an entire traverse composed of multiple intermediate points of interest. This allows for SEXTANT to capture all of the goals of a particular planetary exploration. SEXTANT also allows the explorer to plan concurrent path for two different types of explorers - astronauts on food and transportation rovers. 


\section{Development of SEXTANT}

SEXTANT has been in development in the MIT Man Vehicle Lab since 2001. The first implementation of SEXTANT was called the Geologic Traverse Planner, developed by Carr. ${ }^{2,14}$ With this tool, the user selected ordered waypoints over a terrain digital elevation model and the traverse was drawn between the waypoints as a straight-line path. The traverse was not automatically optimized. Information about the traverse was provided as a total "exploration cost." This took into account both the metabolic cost of carrying out the traverse, and a "sun score", which quantified how good the traverse was with respect to the sun location. Undesirable conditions were when the traverse was directly into or away from the sun, and desirable conditions were when the traverse was perpendicular to the sun. The Geologic Traverse Planner also determined whether the planned traverse violated any constraints set by the user. With this information, the user was required to manually change the traverse waypoints in order to either remove constraint violations or further reduce the exploration cost.

Márquez greatly expanded upon the capabilities of Carr's Geologic Traverse Planner.15-19 The goal of this new system, called the Planetary Aid for Traversing Humans (PATH), was to experimentally investigate the effects of two different levels of automation on a user's performance and situational awareness. In the lower of the two levels of autonomy (called the "passive" automation), the user was responsible for planning paths and the automation simply relayed information. With the higher level of autonomy (called the "active" automation), PATH automatically planned the traverses based on one intermediate waypoint specified by the user. Márquez found that while users with active automation had less costly paths and were able to generate them faster than users with passive automation, they also spent less time performing manual sensitivity analyses. In other words, the users did not modify the path waypoints to see how the costs were affected. This, in turn, led to a loss of situational awareness. SEXTANT currently uses this higher level of autonomy, but also keeps the user involved during the planning process to increase his situation awareness. Even though the path is automatically optimized, the user has specified the Activity Points and has a good knowledge about where the path leads. SEXTANT also displays basic information about the terrain and traverse to increase the user's situational awareness. This data includes the terrain elevation and slope, as well as the traverse distance, time, energy cost, and thermal metrics or rover battery energy.

Development of PATH was continued by Lindqvist, who united the optimization capabilities of PATH with the mapping features of the ArcGIS geographical information system (GIS) software suite. ${ }^{20}$ In this integrated mission planner, ArcGIS served as the interface, allowing the user to view the terrain and specify waypoints directly on a two- or three-dimensional (2D or 3D) terrain map. Once the waypoints were selected, PATH was called as a separate function to optimize the traverse on its exploration cost. The resultant traverse was then displayed on the terrain map. While Lindqvist integrated PATH and ArcGIS together as two separate programs, Essenburg combined the functionality of both into one program, called Pathmaster. ${ }^{21}$ This tool greatly improved the user interface for the PATH algorithms, making it easier to plan traverses and view the desired information. The main Pathmaster interface was a 3D elevation map over which the user could modify obstacles and waypoints and see the generated traverse. This map was created from a matrix of elevations, which for Earth could be exported by GIS tools like ArcGIS. Through the mapping interface, the user could also view elevation and slope information about any point in the terrain model. Furthermore, Pathmaster allowed for traverses to be created for multiple explorers with different physical characteristics (i.e., weight) and waypoints, all over the same terrain. After placing traverse waypoints for all explorers, the paths were optimized on the metric of metabolic cost using an A* (pronounced "A-star") graph search algorithm. The Pathmaster interface has been expanded and modified by this author to serve as the basis of the SEXTANT interface.

\section{Basics of SEXTANT}

SEXTANT is a tool designed for surface explorations of both the Moon and Mars; however, it currently only has the capability to plan lunar and Earth-based traverses (the latter of which are used for field-testing SEXTANT). The extension from lunar to Martian traverses is a simple one; only minor changes and additions must be made. The rest of this paper will discuss the capabilities of the current version of SEXTANT, with a focus on lunar traverses.

\section{A. EVA Representation in SEXTANT}

Any traverse is represented in SEXTANT by three separate components:

- Exploration Objectives, which characterize the activities of the traverse between which the traverse path is created

- Environment Model, which represents the planetary environment in which the traverse is occurring

- Explorer Model, which details the energy consumption and constraints of the explorer performing the traverse 


\section{Exploration Objectives}

Each Exploration Objective is called an Activity Point and consists of three parts: a specific activity, location, and duration. The activity can represent a number of tasks such as sample collection, resource exploration, or terrain scouting, for example. For traverses on Earth, the Activity Point locations can be represented as geographic coordinates in either the Universal Transverse Mercator (UTM) system, or by latitude and longitude. For Activity Points on the Moon, locations can be expressed as indices corresponding to a location on the terrain elevation map. The duration of an Activity Point is the time spent in place at the location performing the specified tasks.

\section{Environment Model}

Regardless of the planet on which the traverse is occurring, the Environment Model consists of three parts. These are the:

- Terrain elevation matrix

- Terrain slope matrix

- Terrain obstacle matrix

When SEXTANT is being used to plan lunar traverses, there is an additional fourth component to the Environment Model:

- Location of sunlit and shadowed areas on the lunar surface

The fundamental component of the Environment Model is a user-inputted elevation map. This map, imported into SEXTANT as a text file, is a matrix specifying the elevation of equally-spaced points on the terrain surface. The distance between points, which is inversely proportional to the number of points, is called the horizontal map resolution. A high resolution (small distance between points) is desirable, because it gives a more precise representation of the terrain. Smaller surface features, like steep cliffs or boulders, can be captured on highresolution maps but may be missed on lower-resolution maps. However, higher-resolution maps result in greater computational complexity and slower processing speed.

The lunar elevation maps used in SEXTANT are derived from data produced by the Lunar Orbiter Laser Altimeter (LOLA) instrument aboard the Lunar Reconnaissance Orbiter (LRO) spacecraft. ${ }^{22-25}$ LRO was launched on June 18, 2009, the culmination of five years of development. Managed by the NASA Goddard Space Flight Center, the goal of LRO's one-year primary mission is to support future human exploration of the Moon. Specifically, LRO's exploratory objectives are to find safe landing sites, locate potential resources, characterize the radiation environment, and demonstrate new technology. To support LRO's goals, LOLA itself has two main objectives. ${ }^{24}$ Firstly, LOLA is measuring the location, direction, and magnitude of surface slopes, as well as the elevation variation (also called the surface roughness). Secondly, LOLA is quantifying the reflectance of the lunar surface (the albedo) to look for the presence of water ice crystals. LOLA is accomplishing these goals by using a $1064 \mathrm{~nm}$-wavelength Nd:YAG laser and detector to measure the distance from LRO's 50-km polar orbit to the lunar surface. The laser beam is split into 5 separate channels that form an " $\mathrm{X}$ " pattern on the lunar surface. Each channel provides a measurement of altitude, surface roughness, and surface brightness. The width of each LOLA ground track is between 50 and $60 \mathrm{~m}$. The $28 \mathrm{~Hz}$ firing rate and the five-beam configuration translate to an average alongtrack spacing of 10-20 m given the spacecraft velocity. After the primary mission is complete, the average track spacing at the equator will be approximately $1 \mathrm{~km}$ on average. In the polar regions, due to the polar inclination of LRO's orbit, the track convergence allows maps with much higher resolution to be derived ( $25 \mathrm{~m}$ or better).

The LOLA data has produced elevation maps of the lunar north and south poles, both of which are used in SEXTANT. Each is a $500 \mathrm{~km}$ by $500 \mathrm{~km}$ gnomonic projection of the most northern or southern $10^{\circ}$ of lunar latitude. A gnomonic projection is one where all great circles on the globe are represented as straight lines on the map ${ }^{\sharp}$. Therefore, a line between two points on the map corresponds to the actual shortest distance between these two points. Both maps of the lunar poles feature a regularly-spaced horizontal grid of points with a resolution of $240 \mathrm{~m}$. This resolution is not as high as is truly desired, as some large and important objects like boulders will undoubtedly be missed. However, this map is only a preliminary elevation map from LOLA, and future data products will have much higher resolution. The flexibility of SEXTANT allows for any matrix of elevations to be inputted, no matter what the resolution.

Once the elevation map has been inputted within SEXTANT, the magnitude of the local slope at all points is calculated using a gradient operation. Like the elevation data, the slope information (a scalar value) can be stored in a matrix of the same size, where each element corresponds to the terrain slope at that particular location. Next,

\# U.S. Geologic Survey, http://egsc.usgs.gov/isb/pubs/MapProjections/projections.html\#gnomonic

6

American Institute of Aeronautics and Astronautics 
SEXTANT determines areas where the explorer is unable to travel due to safety constraints. These areas are called obstacles, and are defined partly by a user-specified maximum slope. Any point with a slope magnitude greater than this limit is regarded as an obstacle. However, this does not completely describe all obstacles. There may be features below the resolution of the map that pose a hazard to explorers. To compensate for this, the user has the ability to manually designate additional obstacles.

The previous three components of the Environment Model - terrain elevation, slope, and obstacles - are required for both Earth- and lunar-based scenarios. The fourth component of the Environment Model, the location of sunlit and shadowed areas, has only been determined for traverses on the Moon. Unlike the other three components, shadowing changes with time; therefore, it would be very time-consuming to create matrices of this information for all points in the terrain map. As a result, shadowing is only determined for points along the traverse after a path has been generated. Shadowing at a point is represented as a value from 0 to 1 , which represents the percentage of the solar disk that is visible from that location. A value of 0 is complete shadow, a value of 1 is in complete sunlight, and a value between 0 and 1 is in partial shadow.

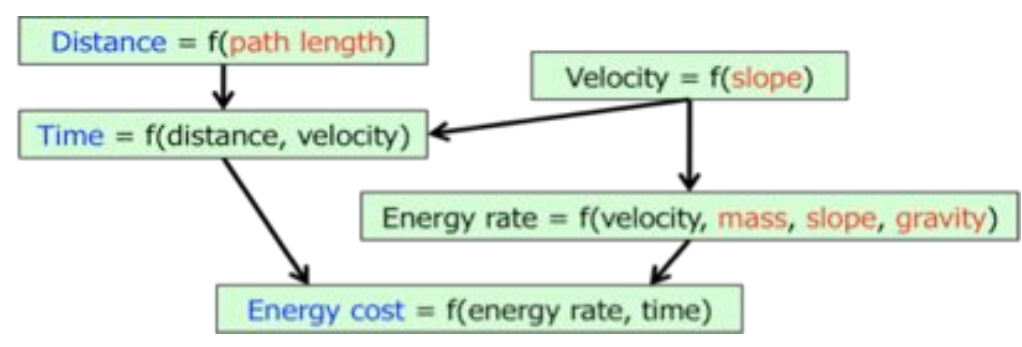

Figure 1. Structure of explorer model within SEXTANT.

Table 1. Velocity equations for suited astronaut explorers. ${ }^{15}$

\begin{tabular}{|c|c|}
\hline Slope, $\alpha\left(^{\circ}\right)$ & Velocity $(\mathrm{m} / \mathrm{s})$ \\
\hline$-20^{\circ} \leq \alpha<-10^{\circ}$ & $0.095 \cdot \alpha+1.95$ \\
$-10^{\circ} \leq \alpha<0^{\circ}$ & $0.06 \cdot \alpha+1.6$ \\
$0^{\circ} \leq \alpha<6^{\circ}$ & $-0.2 \cdot \alpha+1.6$ \\
$6^{\circ} \leq \alpha<15^{\circ}$ & $-0.039 \cdot \alpha+0.634$ \\
$15^{\circ} \leq \alpha \leq 20^{\circ}$ & 0.05 \\
\hline
\end{tabular}

Table 2. Metabolic rate equations for suited astronaut. ${ }^{13}$

\begin{tabular}{|c|c|}
\hline \multicolumn{2}{|c|}{ Metabolic Rate $(\mathrm{W})=W_{\text {level }}+W_{\text {slope }}$} \\
\hline \multicolumn{2}{|c|}{$W_{\text {level }}=[3.28 \cdot m+71.1] \cdot[0.661 \cdot \mathrm{v} \cdot \cos (\alpha)+0.115]$} \\
\hline Slope, $\alpha$ & $W_{\text {slope }}$ \\
\hline$\alpha=0^{\circ}$ & 0 \\
\hline$\alpha>0^{\circ}$ & $3.5 \cdot m \cdot g \cdot v \cdot \sin (\alpha)$ \\
\hline$\alpha<0^{\circ}$ & $2.4 \cdot m \cdot g \cdot v \cdot \sin (\alpha) \cdot 0.3^{|\alpha|}$ \\
\hline
\end{tabular}

Table 3. Energy rate equations for transportation rovers. ${ }^{14}$

\begin{tabular}{|c|c|}
\hline \multicolumn{2}{|c|}{ Energy Rate $(\mathrm{W})=W_{\text {level }}+W_{\text {slope }}+P_{e}$} \\
\hline \multicolumn{2}{|c|}{$W_{\text {level }}=0.216 \cdot v \cdot m$} \\
\hline Slope, $\alpha$ & $W_{\text {slope }}$ \\
\hline$\alpha=0^{\circ}$ & 0 \\
$\alpha>0^{\circ}$ & $0.02628 \cdot m \cdot \alpha \cdot(\mathrm{g} / 1.62) \cdot v$ \\
$\alpha<0^{\circ}$ & $-0.007884 \cdot m \cdot \alpha \cdot(\mathrm{g} / 1.62) \cdot v$ \\
\hline
\end{tabular}

\section{Explorer Model}

The Explorer Model represents the explorer carrying out the traverse. It also comprises the metrics on which the most efficient path can be determined: the path distance, the travel time, and the energy cost to travel along a path. Whichever of these is being used is called the cost function. The efficiency of a traverse increases as the distance, time, or energy cost required to travel it decreases. SEXTANT contains explorer models for human astronauts and transportation rovers, both of which will be heavily involved in future planetary and lunar exploration. These two models have the same structure, which can be seen in Fig. 1. The three metrics of distance, time, and energy cost shown in blue in Fig. 1 are all functions of four parameters, shown in red.

For human astronauts, the explorer velocity parallel to the terrain surface is solely a function of the slope. The relation for human astronauts was developed by Márquez from data on the Apollo 14 mission compiled by Waligoria and Horrigan. ${ }^{15,26}$ This is detailed in Table 1.

The astronaut energy rate is a function of his velocity and mass, the terrain slope and the planetary gravitational acceleration. ${ }^{13}$ The explorer mass captures the astronaut's body, the space suit, and any extra equipment or samples being carried. The energy rate is broken up into two components: the energy required for locomotion over a level surface, and the energy required to travel up or down a slope. As a result, the velocity given in Table 1 (measured 
parallel to the terrain surface) must be broken into horizontal $\left(v^{\bullet} \cos (\alpha)\right)$ and vertical $\left(v^{\bullet} \cos (\alpha)\right)$ components. The metabolic energy rate is related to these parameters by the equations set forth in Table 2 .

Unlike astronauts, the rover velocity is assumed to be a constant $15 \mathrm{~km} / \mathrm{hr}(4.17 \mathrm{~m} / \mathrm{s})$ over all traversable terrain. Even so, the transportation rover energy rate does vary with the terrain slope. The energy rate is a function of the same parameters as the astronaut's metabolic rate: the rover velocity and mass, and the terrain slope and planetary gravitational acceleration. $^{14,27}$ The rover mass consists of the rover structure and all payload, including the astronauts. Because the equations were developed from historical data on the LRV, the equations are normalized to lunar gravity $\left(1.62 \mathrm{~m} / \mathrm{s}^{2}\right)$. The energy rate is related to these parameters by the equations set forth in Table 3 .

As with the astronaut explorer model, there are components of the energy for traveling across flat terrain and for up- or downhill. Unlike suited astronauts, there is an additional component of energy, the collection of all other electronic components in the rover. This exists even when the rover is stationary, and is assumed to be a constant $1500 \mathrm{~W}$ throughout the traverse. ${ }^{28}$

\section{B. Optimization Algorithm}

SEXTANT employs an A* (pronounced "A-star") graph search algorithm to compute the most efficient path between Activity Points, regardless of which metric is being used as the cost function. This algorithm, developed by Hart et al., optimizes the path between two points on the metric of lowest cost. ${ }^{29}$ In the $\mathrm{A}^{*}$ search, all points in the terrain map are represented as "nodes". The A* search is called multiple times for a traverse, each time exploring a number of paths through different nodes to find the most efficient path between two consecutive Activity Points. Once an Activity Point is reached as the goal of one A* search, it becomes the starting node for the subsequent $\mathrm{A}^{*}$ search. For specific details on how each instance of the $A^{*}$ search is carried out, please refer to Ref. 30 .

A heuristic function dictates the behavior of the $\mathrm{A}^{*}$ search in finding the least costly path, and is a trade-off between speed and accuracy. A low heuristic will give a more accurate result, meaning that the path cost will be closer to the true least-costly path between two Activity Points. In fact, as long as the heuristic function is admissible, meaning that it underestimates the true cost of moving from any node to the goal Activity Point, the A* search is guaranteed to find the true least-costly path. However, the search will explore more nodes in finding this path, and will take longer to complete. On the other hand, a high heuristic will run faster but give less accurate results.

SEXTANT's heuristic function is admissible, and computes the minimum number of lateral and diagonal steps that must be taken between any node and the goal Activity Point. The number of steps in each direction must be multiplied by their respective movement costs. Because the heuristic guarantees the minimum number of steps between any node and the goal Activity Point, these costs must also give the lowest energy possible. This will ensure that the heuristic does not overestimate the actual cost to travel to the goal Activity Point, making the heuristic admissible. For both astronauts and transportation rovers, there is a specific terrain slope at which the energy cost per unit distance is a minimum. This slope is $-5.85^{\circ}$ for astronauts on Earth, $-4.2^{\circ}$ for astronauts on the Moon, and $0^{\circ}$ for rovers on the Moon.

\section{SEXTANT Graphical User Interface}

The graphical user interface (GUI) for SEXTANT allows the user to easily interact with the traverse representation and plan explorations across the planetary surface. This GUI consists of two main parts. First, there are the input menus, where the user can specify many parameters of the traverse representation, astronaut thermal model, and rover power model. These input menus also give the user the ability to add additional explorers - either astronauts or transportation rovers - each with their own unique parameters. These input menus are encountered upon initializing SEXTANT, but can be returned to at any point in the planning process. Secondly, there is the 3D mapping interface, which is the main interface for SEXTANT. This GUI allows the user to specify Activity Points on a terrain map, run the most efficient path calculation, and view the resultant traverse path and information calculated by the A* algorithm.

\section{3D Mapping Interface}

The 3D mapping interface shows the selected terrain and obstacles as dictated by the user-specified maximum slope. In Figs. 2 and 3, which show traverses around the lunar south pole, the accessible lunar surface is colored grey, while the red areas are obstacles as defined by the maximum slope. To adjust the obstacles, the user selects the Terrain button at the top of the interface, chooses a tool size, and then clicks on the map to place a new obstacle or holds the shift key and clicks to remove an existing one. These areas are modified in the obstacle matrix and displayed on the terrain map. Activity Points can be placed on the terrain map simply by clicking the desired 
location on the terrain map, and must be specified in the order they are to be visited. The user can generate unique paths for as many explorers as have been defined. Each set of explorer Activity Points is displayed in a different color to easily distinguish between them. In Fig. 2, there are two explorers, the first marked in blue, and the second in yellow. If an explorer is to remain at any of the Activity Points for a period of time, the durations can be specified by clicking on the Stay at AP button once the traverse has been created.

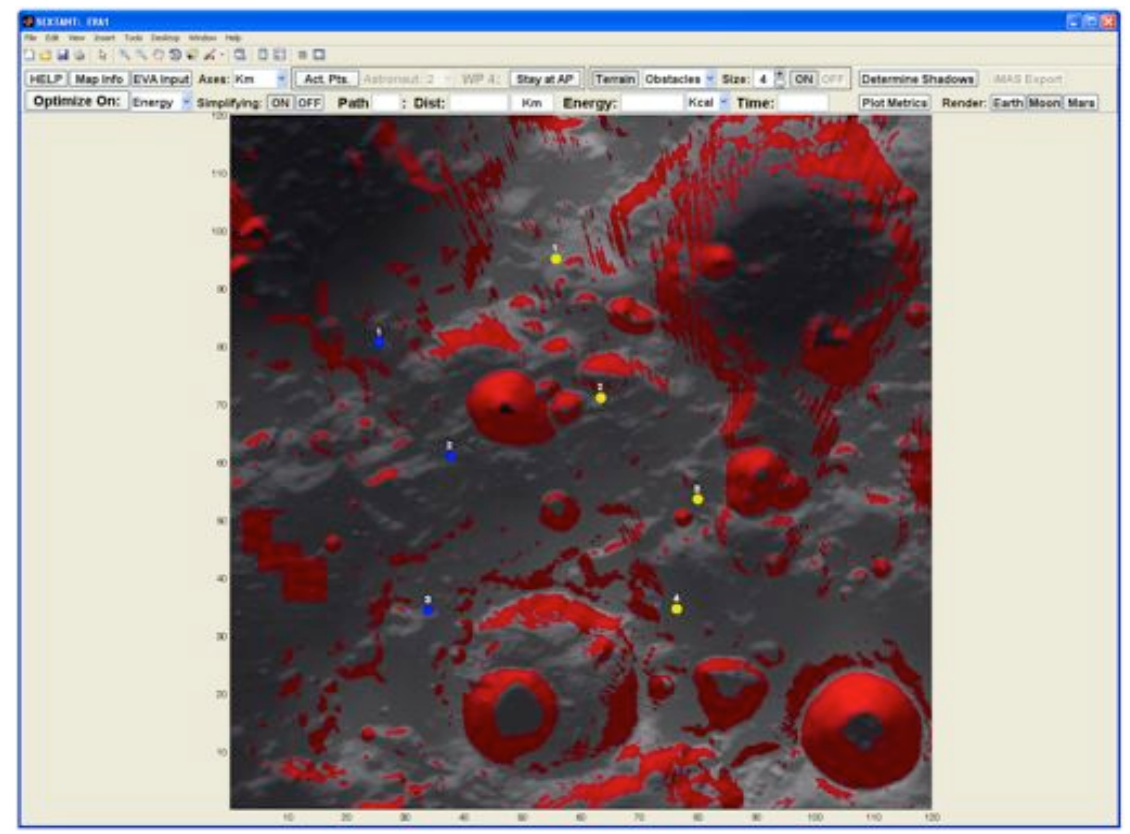

Figure 2. Activity Points specified for two explorers.



Figure 3. 3D view of generated traverse paths for two explorers. 


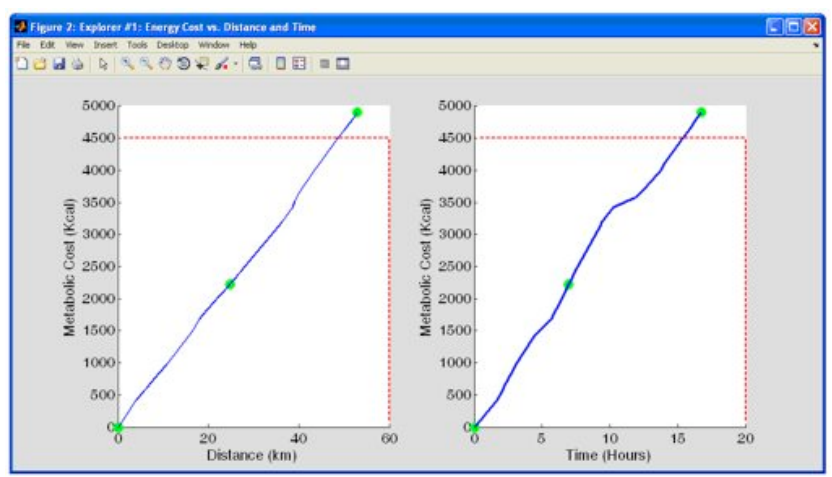

Figure 4. Metabolic cost of traverse with respect to distance and time.

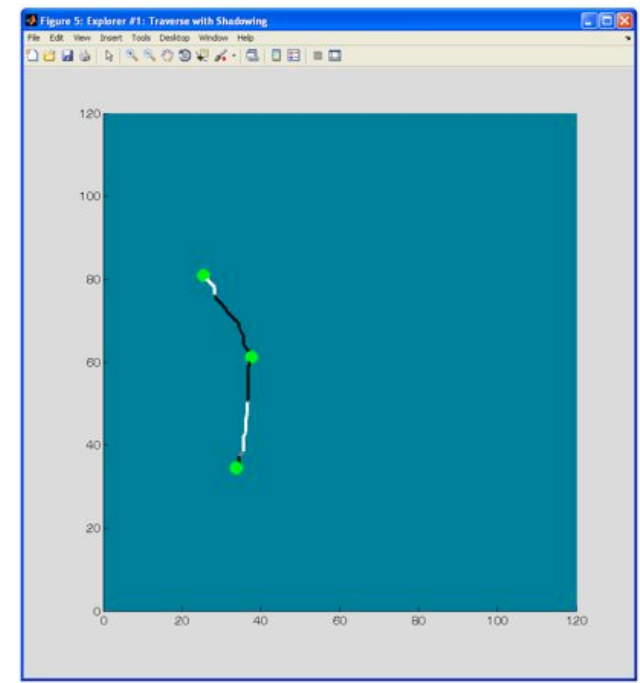

Figure 5. Shadowing along traverse.



Figure 6. Return-home path for blue explorer.
Once the user has completely specified the Activity Points for all explorers, he can click the Optimize On button to generate the most efficient traverse paths. The user also has the ability to change the metric on which this most efficient path is determined, be it the traverse distance, time, or energy cost. If any Activity Point is completely surrounded by obstacles and entirely inaccessible, SEXTANT returns an error and will not continue the path calculations. Each explorer's traverse path is displayed on the terrain map as a series of straight lines connected by intermediate Path Points, where the explorer changes direction. These paths are marked in the same color as the explorer's Activity Points for easy identification. Figure 3 shows traverse paths for the two astronauts. The original user-selected Activity Points have been highlighted by underlying green circles.

Beyond determining the most efficient path, SEXTANT calculates the value of the three possible cost functions at each Path Point, regardless of the explorer type. Specifically, these metrics are the:

1. Cumulative path length from the habitat (the first Activity Point) to the Path Point

2. Time to travel from the habitat to the Path Point along the path

3. Energy cost to travel from the habitat to the Path Point along the path

All three metrics are computed for each Path Point, and the total values for the entire traverse are displayed at the top of the 3D mapping interface. The cumulative energy cost is also plotted against the traverse time and distance, to give an overall picture of how this changes throughout the traverse (Fig. 4). The sections of the graph with a steeper slope represent more difficult portions of the traverse - where the energy cost is increasing at a faster rate. Areas of the traverse with a lower slope on the graph are easier to travel in comparison. The graphs also display user-inputted time, distance, and energy constraints, indicated by dashed red lines. These show whether or not the traverse is valid, and if it is not, what needs to be changed. In Fig. 4, it can clearly be seen that the EVA of Astronaut 1 is within the distance and time constraints, but exceeds the metabolic cost constraint. The traverse must be re-planned.

SEXTANT also has the ability to compute shadowing-related metrics for the explorers. These are different for astronauts and transportation rovers, and are the outputs of either the astronaut thermal model or the rover power model. For astronauts, the following additional metrics are computed for each Path Point: 
4. Cumulative mass of water needed to replenish ice sublimated away for cooling during the traverse from home to the Path Point

5. Cumulative heater energy required for heating during the traverse from home to the Path Point

Instead of these, transportation rovers have only one additional metric, once again computed for each Path Point:

4. Battery energy level at the current Path Point

After this information is calculated, it is displayed graphically with respect to the traverse distance or time. A graphic of the traverse is also displayed in Fig. 5, showing which areas are in sunlight, and which are in shadow. In this figure, the white areas are where the solar disk is completely visible (full sunlight), the black areas are where the solar disk is completely hidden (full shadow), and the grey areas are where the solar disk is only partially visible.

SEXTANT is also able to calculate return-home paths, an important capability. These represent emergency "walk-back" situations, when the astronaut must return to the habitat quickly. In the 3D mapping interface, the user right-clicks on any point along a path (not necessarily a Path Point) to determine the most efficient path back to the habitat from this location. This path is displayed on the terrain with a dashed line, and the corresponding path length, time of travel, metabolic cost, shadowing, and thermal or power metrics are displayed. Figure 6 shows a returnhome path for Astronaut 2 (notation 1). SEXTANT also calculates the shadowing along a return-home path, and the specific shadowing-related metrics for the explorer. If the return-home path begins at a Path Point, where the shadowing-related metrics are known for the original traverse, the metrics for the return-home path will begin with these values. This accurately represents an astronaut traveling along the original path to a point, and then heading home along the return-home path. If the return-home path does not begin at a Path Point, the shadowing-related metrics of the return-home path are determined absolutely - from 0 for the astronaut thermal metrics, and from full battery for the rover power metric.

The ability to determine return-home paths is very important during pre-traverse contingency planning and emergencies en-route. Explorers can only carry a limited supply of consumables, which imposes a strict limit on the traverse length, time, and difficulty. As an explorer is traveling across the lunar or planetary surface, he can calculate return-home paths for future points along the traverse. Knowing the costs of these return-home paths allows him to determine the "point of no return", past which he would not have enough consumables to safely return home. This allows the explorer to maximize the productivity of the traverse while remaining safe.

\section{Real-time Navigation}

The framework for an implementable, user-centric SEXTANT interface has been laid by integrating SEXTANT with the Individual Mobile Agents System (iMAS), developed at NASA Ames Research Center. ${ }^{31-39}$ This combined system gives the astronaut user an auditory interface through which he can input, store, and retrieve information about the EVA. This interface does not contain a push-to-talk button; rather, iMAS is constantly listening to the user and responds to recognized commands. This allows both the astronaut's hands to be free for work during the EVA. In the future, this auditory interface can be combined with a visual interface to serve as a tool that astronauts could use for real-time planning and re-planning while on the surface of the Moon or Mars. Currently, SEXTANT and iMAS have been integrated to provide real-time navigation along a traverse path planned in SEXTANT. As iMAS requires a GPS device to track the astronaut, this integrated system can only be used for Earth-based traverses at this time. With the development of a guidance system for the Moon and Mars, iMAS could easily be extended to these explorations.

iMAS has a number of capabilities to assist astronauts during EVA. It can store multiple named locations, and at any time the astronaut can ask for the distance and heading to a location with respect to his current position and bearing (known through a GPS device). The astronaut accomplishes this by speaking a query such as, "Where is Work Site 2?" iMAS then responds with a phrase such as, "Work Site 2 is 20 meters, $45^{\circ}$ to the left." iMAS can also manage an ordered list of activities, each modeled by a location (or locations, if the activity involves movement) and duration. The astronaut can verbally start any activity, after which iMAS keeps track of the elapsed time. iMAS issues an auditory alert once the astronaut approaches the end of the scheduled activity duration. To easily record scientific data and observations gathered during an EVA, the astronaut can record voice notes at any time to be reviewed later. Recording a voice note is faster and easier than writing a physical note, especially while wearing space suit gloves. Finally, iMAS can monitor the astronaut's biomedical parameters by connections to the Life Support, Exploration Guidance Algorithm and Consumable Interrogator (LEGACI) developed by Kuznetz at Johnson Space Center. ${ }^{40}$ LEGACI uses a collection of biosensors located within the space suit to collect information about the EVA status, consumables, and astronaut performance data. iMAS allows the astronaut to access this information in real time, by querying about the amount of remaining consumables, metabolic parameters, or the space suit status.

11

American Institute of Aeronautics and Astronautics 
Within the integrated system, the SEXTANT interface is used to specify Activity Point locations spatially on a terrain map of the Earth's surface. Activity Points can be placed and modified on the 3D mapping interface, and the path can be optimized for distance, time, or energy cost. Once the traverse is deemed satisfactory, it can be imported into iMAS as an .xml file. This file creates a location for each Path Point and activities that encapsulate movement between each set of consecutive Path Points. These activities also specify the duration of each segment, as computed by SEXTANT. This allows the astronaut to check and see if he is traveling faster or slower than expected for a certain traverse stage. SEXTANT also creates a gps.dat file with a list of latitude and longitude coordinates along the traverse at a user-specified time interval. If iMAS has no connection to a real GPS receiver, it can read the astronaut's position in time from this file. This allows for the user to simulate an astronaut following the path planned in SEXTANT - a "virtual field test."

Once the traverse has been imported into iMAS, the explorer is tracked through a GPS signal as he follows the planned path. In lieu of an actual GPS signal, iMAS can read the astronaut's position off the gps.dat file produced by SEXTANT. At any point along the traverse, the astronaut can ask iMAS for the heading and direction to stored Path Points. This information allows the user to follow the most-efficient path between Activity Points, as planned in SEXTANT. iMAS effectively takes the information generated by SEXTANT and implements it in a real-world situation. In the future, an astronaut on EVA will be able to plan and re-plan paths through a small SEXTANT interface mounted on the space suit (as a wrist-based device or as part of a heads-up display, for example). He will then be able to import the plan to iMAS and carry it out. This will ensure that the abilities of SEXTANT are accessible during actual EVAs.

\section{Shadowing}

The ability of SEXTANT to determine shadowed and sunlit areas on the lunar surface is one of its most important functions. Knowing the shadowing allows the astronaut's thermal load and the power usage and generation of transportation rovers to be computed. Both of these directly influence the explorer's consumables, which are the main limiting factors of the traverse. Before a traverse is begun, an astronaut must ensure that he will be able to complete it without running out of water or power.

Once a traverse has been planned across the lunar surface, SEXTANT parses the traverse into a series of stages between consecutive Path Points and computes the astronaut thermal load or rover power generation and consumption over each individual stage. In order to do this, the shadowing must be determined at each Path Point. SEXTANT accounts for the movement of the sun during the traverse by calculating its position with a certain time step. This time step is defaulted to every 2 hours, representing a $1^{\circ}$ change in the sun position. A smaller time step will give a more accurate result, but increase the computational time. If the value of the time step is more than twice the travel time for the traverse, then the sun will remain in the same position for the entire traverse. The time at which the explorer arrives at each Path Point is rounded to the closest multiple of this time step. This "binning" gives a series of unique sun times at which the sun position must be calculated. The position of the sun is calculated at each of these times with respect to the Moon's center of mass through the use of the NASA Jet Propulsion Lab SPICE toolkit for MATLAB (called MICE) ${ }^{\S}$. SPICE is a program that gives ephemeris data for any target body in the solar system. Loosely, ephemeris data is defined as the position of a target in the sky as seen from another body, as a function of time. MICE is a version of SPICE that is fully contained in MATLAB; no outside connections are required. Because MICE returns the sun position with respect to the Moon's center of mass, SEXTANT performs coordinate transformations to get the elevation and direction of the sun from each Path Point at the correct time.

After the sun position is known for all Path Points, SEXTANT must determine whether or not it is visible at each particular time. This is accomplished using the horizon method, used for the Moon by Garrick-Bethell et al. with ground-based radar data ${ }^{41}$ and more recently by Mazarico et al. with the LOLA data. ${ }^{42,43}$ Mazarico et al. distinguish two distinct steps in this method. First, a database is created of 720 separate horizon maps that each shows the elevation of the horizon in a certain direction for all points on the terrain map. Each of these horizon maps is in a direction that varies $0.5^{\circ}$ from the surrounding maps. This step is very time consuming - on the order of a week using fifteen MacPro computers - but must only be performed once. The second step in the horizon method requires referencing these horizon elevation maps to determine whether or not the sun is visible in a certain direction for a Path Point. If the sun direction is not an exact interval of $0.5^{\circ}$, then linear interpolation is used between the adjacent horizon elevation maps. Because the lunar surface elevation map is in gnomonic projection, the sun is in the same direction for each point on the map at one particular time. So, only one horizon elevation map must be referenced for the entire set of Path Points at the same unique sun position time. Once the horizon elevation is calculated for

$\S \S$ Available from the Navigation and Ancillary Information Facility at http://naif.jpl.nasa.gov/naï/index.html. 
each Path Point, it is compared to the elevation of the sun. As seen from the surface of the Moon, the solar disk has an apparent diameter of 32 arcminutes $\left(0.52^{\circ}\right)$. Because the sun is not a point source, there are three possible conditions for the shadowing at each Path Point:

- The sun is fully visible (complete sunlight)

- The sun is partially visible (partial shadow)

- $\quad$ The sun is not visible at all (complete shadow)

To account for all conditions, SEXTANT computes the percentage of the solar disk visible as a value from 0 to 1 and stores this for each Path Point. The shadowing for each path stage is determined as the average of the sun visibility percentages for the two Path Points at either end. This information can be used to compute the thermal load on astronauts and the power consumption and generation of transportation rovers for each path stage, and for the entire traverse together.

\section{A. Astronaut Thermal Model}

Knowing the thermal load on an astronaut throughout an EVA is important because it directly relates to the amount of consumables required for the traverse. For astronauts, the two main thermal consumables of concern are the mass of water required to produce ice for cooling by sublimation and the amount of energy required to run the space suit heater. The mass of water for sublimation and power system that can be carried by an astronaut are limited by the volume and mass constraints of the space suit's life support system. As such, it is important to be able to accurately estimate the amount of these consumables that the astronaut will require for a traverse. This ensures that the astronaut will have enough thermal control to complete the EVA and safely return to the habitat.

In the SEXTANT astronaut thermal model, there are five sources of heat transfer to and from the space suit. There are two internal sources: heat from the inefficiency of astronaut work and the space suit electronics waste heat. There are three external heat sources: direct sun radiation, radiation between the space suit and the lunar surface, and radiation between the space suit and deep space. The external heat reaches the astronaut by conduction through the space suit, which is dictated by the temperature of the outer surface of the space suit. The sign convention used for the heat flux is positive for heat entering the space suit, and negative for heat leaving the space suit.

\section{Heat Transfer from Internal Sources}

The energy consumed by the astronaut when carrying out an EVA (Table 2) is used in a number of different processes by the astronaut's body. A portion is the basal metabolism, which is used for basic physiological processes like breathing, pumping blood, and digestion. ${ }^{44}$ An additional portion is used to do useful work in moving the astronaut across the terrain. The remainder of the energy consumed is transformed into heat, which is both stored in the body and released to the environment. Equation (1) details these components that make up the total energy consumption $^{44}$. Basal metabolism is absent, because these equations deal with work, and not energy.

$$
\dot{Q}_{m}=\dot{W}_{w}+\dot{W}_{w c}+\dot{W}_{w r}+\dot{W}_{w s}+\dot{Q}_{n}+\dot{Q}_{s}
$$

A few assumptions are made to simplify this model within SEXTANT. First of all, the work done to move the astronaut's body over a flat surface $\left(\dot{W}_{w r}+\dot{W}_{w c}\right)$ is assumed to be $0 \mathrm{~W}$, as is the body heat storage. The latter assumes that all of the heat generated by the inefficiency of work is transferred to the space suit. In reality, there is heat flow from the muscles to the surrounding tissue and blood, and only some of the heat generated by the inefficiency of work is released to the space suit atmosphere by respiration, conduction, and radiation. ${ }^{45}$ Because of $^{\circ}$ this, the amount of heat from the astronaut's metabolic workload is somewhat overestimated in SEXTANT.

The external mechanical work is the work required to change the potential energy of the astronaut by moving upor downhill. The amount of energy used for external work at a constant speed can be quantified in a term called the mechanical efficiency. ${ }^{46}$ This is equal to the mechanical work performed by the astronaut divided by his total energy consumption.-When the terrain slope is $0^{\circ}$, the efficiency is 0 . As the terrain slope increases (walking uphill), so does the mechanical efficiency, until it reaches a plateau of 0.25 at a slope of $20^{\circ}$. As Margaria states, "this is the characteristic mechanical efficiency of muscle performing positive work, as is also found in isolated muscle preparation". "Whenever the astronaut is walking downhill, the efficiency is negative. This occurs because the mechanical work being performed is also negative - the astronaut is traveling in the same direction as the gravitational force. As a result, additional heat from the negative work is being absorbed by the astronaut's muscles. It is important to note that energy is still being consumed because the leg muscles are still active. Margaria's data is approximated in SEXTANT by the piecewise function shown in Table 4.

13

American Institute of Aeronautics and Astronautics 
Table 4. Function describing astronaut mechanical efficiency.

\begin{tabular}{|c|c|}
\hline Slope, $\alpha$ & Mechanical Efficiency, $E$ \\
\hline$\alpha<-12^{\circ}$ & -1.2 \\
$-12^{\circ} \leq \alpha<-10^{\circ}$ & $0.15 \cdot \alpha+0.6$ \\
$-10^{\circ} \leq \alpha<-5^{\circ}$ & $0.1 \cdot \alpha+0.1$ \\
$-5^{\circ} \leq \alpha<0^{\circ}$ & $0.08 \cdot \alpha$ \\
$0^{\circ} \leq \alpha<5^{\circ}$ & $0.025 \cdot \alpha$ \\
$5^{\circ} \leq \alpha<10^{\circ}$ & $0.015 \cdot \alpha+0.05$ \\
$10^{\circ} \leq \alpha<20^{\circ}$ & $0.005 \cdot \alpha+0.15$ \\
$20^{\circ} \leq \alpha$ & 0.25 \\
\hline
\end{tabular}

Carr and Newman suggest that the muscles are optimized for the Earth's gravitational field, and that their efficiency decreases as the planetary gravity decreases. ${ }^{44}$ Therefore, the efficiency found from Margaria's data must be multiplied by a scaling factor called $E_{\text {planet }}$, which tells how much the muscle's efficiency decreases relative to Earth. Carr and Newman found that $E_{\text {planet }}$ for Mars is 0.78 and $E_{\text {planet }}$ for the Moon is $0.48 .^{44} E_{\text {planet }}$ for Earth is, of course, 1 . This gives the final equation for the external mechanical work as:

$$
\dot{W}_{w}=E_{\text {planet }} \cdot E \cdot \dot{Q}_{m}
$$

The work used to move the space suit is required to compress the oxygen within the suit and deform the suit material, and is a significant component of the total work done by the astronaut. Carr and Newman noted that the energy required to move the space suit is $80 \%$ of the total non-basal metabolic rate - the amount of energy consumed for useful work. ${ }^{44}$ Because Eq. (1) requires work and not energy, the energy required to move the space suit must be multiplied by both the characteristic mechanical efficiency for muscles, given by Margaria as $0.25{ }^{46}$ and by $E_{\text {planet. }}$ This gives:

$$
\dot{W}_{w s}=0.2 E_{\text {planet }}\left(\dot{Q}_{m}-\dot{Q}_{b}\right)
$$

The basal metabolic rate can be determined from the metabolic rate equations in Table 2, with a velocity of 0 . Combining Eqs. (1), (2), and (3), the total heat loss from the astronaut to the space suit is:

$$
\dot{Q}_{n}=\dot{Q}_{m}\left(1-E \cdot E_{\text {planet }}-0.2 E_{\text {planet }}\right)+0.2 E_{\text {planet }} \dot{Q}_{b}
$$

The second component of internal heat is the space suit electronics waste heat. This is the heat that is produced by the inefficiencies of electrical components in the space suit. Ideally, the space suit electronics waste heat should vary depending on the astronaut's activities at a certain time. As more battery power is required for life support, thermal control, communications, and other components, the electronics waste heat will increase. However, SEXTANT does not have the ability to model the astronaut's changing activity level for a traverse. The electronics waste heat is therefore considered as a user-specified constant value throughout the entire EVA.

\section{Heat Transfer to or from External Sources}

The direct sun radiation is the largest source of heat into the space suit, when present. This heat flux is determined by the equation:

$$
\dot{Q}_{\text {sun }}=V_{\text {sun }} \alpha_{\text {sun }} A_{\perp} I
$$

The space suit area perpendicular to the sun is used because solar radiation is only contacting this area, and not the entire surface of the space suit. In SEXTANT, $A_{\perp}$ is assumed to be half of the total space suit surface area. The surface area is approximated with the Mosteller formula for body surface area, using the astronaut's suited height and mass. ${ }^{47}$

Radiation between the space suit and the environment is governed by the equation:

$$
\dot{Q}_{\text {suit-env }}=\sigma \varepsilon_{\text {suit }} \varepsilon_{\text {env }} A_{\text {suit }} F_{\text {suit -env }}\left(T_{\text {env }}^{4}-T_{\text {suit }}^{4}\right)
$$

In SEXTANT, the space suit thermally interacts with two different parts of the environment (besides the sun, which has already been accounted for): the lunar surface and deep space. As a result, Eq. (6) is applied twice with 
slightly different parameters. If $\dot{Q}_{\text {suit-env }}$ is negative, heat is leaving the space suit to the environmental component, and if $\dot{Q}_{\text {suitenv }}$ is positive then heat is entering the space suit from the particular environmental component. $A_{\text {suit }}$ is the total surface area of the space suit, and not just half as was seen in Eq. (5). This is because the $A_{\perp}$ in Eq. (5) had to account for the area of the suit that was involved in the heat transfer. In Eq. (6), this is accounted for by the view factor between the suit and the environmental component, instead of by the area. The view factor shows the percentage of radiation leaving the space suit that is striking the environmental component. It is a general property of view factors that they range in value from 0 to 1 , and that all of the view factors from any object sum to 1 . Within SEXTANT, the only two view factors are between the space suit and the lunar surface and between the space suit and deep space. For simplification, each of these is assumed to be 0.5. Finally, Eq. (6) involves the temperature of the space suit and the specific component of the environment. The temperature of deep space is assumed to be $3 \mathrm{~K}$ $\left(-270^{\circ} \mathrm{C}\right)$. The temperature of the lunar surface is a function of the sun visibility, and can be described by the equation:

$$
T_{\text {Moon }}=250 \cdot V_{\text {sun }}+123
$$

The temperature reaches a maximum of $373 \mathrm{~K}\left(100^{\circ} \mathrm{C}\right)$ in complete sunlight and $123 \mathrm{~K}\left(-150^{\circ} \mathrm{C}\right)$ in complete shadow $^{27}$. This equation is only an approximation, as it does not directly take into account the amount of time the lunar surface has been in shadow or sunlight. This is important, because the lunar surface does not heat up and cool down instantly as it transitions between shadow and sunlight. Rather, there is a heating or cooling process that takes some time. However, the current version of SEXANT neglects this transient effect to simplify the model.

The external temperature of the space suit, $T_{\text {suit }}$ in Eq. (6), constantly changes with time. However, in SEXTANT, it is assumed to remain constant for each individual stage of the traverse and only change at Path Points between stages. This allows for the radiation heat transfer in Eq. (6) to be easily calculated for a stage. The temperature of the space suit at the end of a stage (and consequently the beginning of the next stage) is in turn dictated by the total environmental heat flux during that stage by the equation:

$$
T_{\text {suit }, 2}=\frac{\dot{Q}_{\text {env }} t}{m_{\text {suit }} c_{p, \text { suit }}}+T_{\text {suit }, 1}
$$

The temperature of the space suit at the beginning of first stage of the traverse is assumed to be $300 \mathrm{~K}\left(27^{\circ} \mathrm{C}\right)$, the same temperature of the habitat from which the astronaut emerges. It is also assumed that the thermal control system works to keep the atmospheric temperature of the space suit at a constant $300 \mathrm{~K}\left(27^{\circ} \mathrm{C}\right)$.

If the environmental conditions (dictated solely by the shadowing, $V_{\text {sun }}$ ) remains constant for long enough, the external space suit temperature will reach a thermal equilibrium temperature $\left(T_{\text {equil }}\right)$ where the environmental heat into the space suit's external surface equals the heat radiated from this surface to the environment. This temperature is governed by Eq. (9).

$$
T_{\text {equil }}=\left(\frac{V_{\text {sun }} \alpha_{\text {sun }} A_{\perp} I+\sigma \varepsilon_{\text {suit }} \varepsilon_{\text {Moon }} A_{\text {suit }} F_{\text {suit-Moon }} T_{\text {Moon }}^{4}+\sigma \varepsilon_{\text {suit }} A_{\text {suit }} F_{\text {suit-space }} T_{\text {space }}^{4}}{\sigma \varepsilon_{\text {suit }} \varepsilon_{\text {Moon }} A_{\text {suit }} F_{\text {suit-Moon }}+\sigma \varepsilon_{\text {suit }} A_{\text {suit }} F_{\text {suit-space }}}\right)^{1 / 4}
$$

Whenever the astronaut is in complete shadow, the thermal equilibrium temperature is $102.8 \mathrm{~K}$, and whenever the astronaut is in complete sunlight, the thermal equilibrium temperature is $331.6 \mathrm{~K}$. The external temperature of the space suit always moves towards the current thermal equilibrium temperature over each traverse stage. The rate of temperature change is proportional to the difference between the thermal equilibrium and external space suit temperatures, each to the fourth power $\left(T_{\text {equil }}^{4}-T_{\text {suit }}^{4}\right)$.

The external temperature of the space suit determines the amount of heat conduction through the space suit wall during a stage of the traverse. This is the amount of external heat that contributes to the thermal load on the astronaut. This heat flux will be either positive or negative (into or out of the space suit, respectively), depending upon the relative temperature gradient across the space suit. It is described by the equation:

$$
\dot{Q}_{\text {ext }}=C A_{\text {suit }}\left(T_{\text {suit }}-T_{\text {atm }}\right)
$$




\section{Computation of Total Space Suit Heat Flux}

The heat flux that must be balanced out by the thermal control system is the sum of the external heat flux conducted through the space suit and the two internal heat fluxes:

$$
\dot{Q}_{\text {total }}=\dot{Q}_{n}+\dot{Q}_{\text {waste }}+\dot{Q}_{\text {ext }}
$$

The internal heat fluxes are always positive, as they can only transfer heat into the space suit environment. The sign of the external flux will change depending upon the environmental conditions. The total heat flux can be multiplied by the stage time, which gives the total heat into or out of the space suit during the stage.

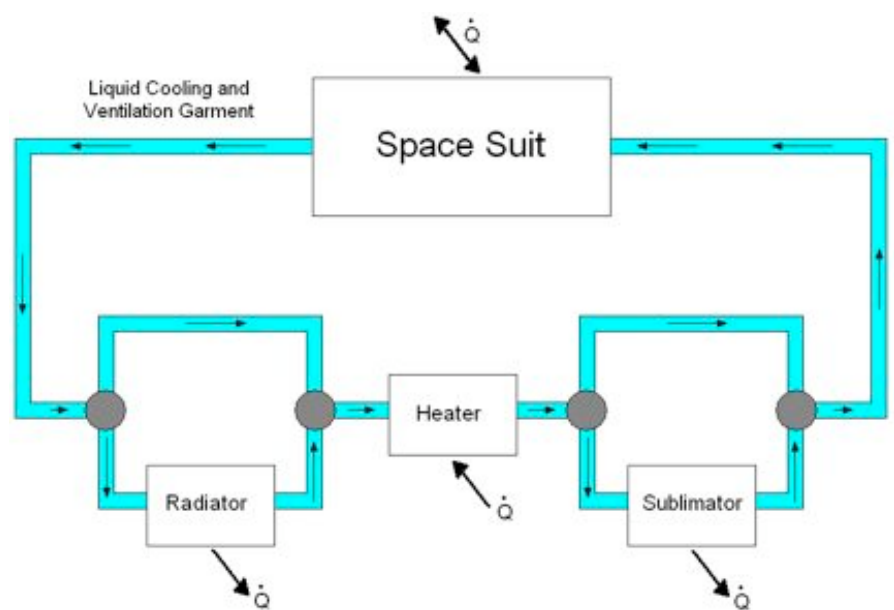

Figure 7. Diagram of space suit thermal control system.

\section{Space Suit Thermal Control System}

In order to keep the space suit at a constant temperature of $300 \mathrm{~K}\left(27^{\circ} \mathrm{C}\right)$, the total space suit heat gain or loss must be balanced by the heat rejection or addition of the thermal control system. If heat is being transferred out of the space suit, then an equal amount of heat must be added to the system. If heat is being transferred into the space suit, then an equal amount must be removed from the system. This is accomplished through the space suit thermal control system, depicted in Fig. 7.

The thermal control system consists of three main components that can either reject heat from or add heat to the system. First of all, a radiator is used to reject an initial amount of heat to deep space. Current space suits do not contain a radiator, but SEXTANT leaves the possibility open for future space suits. To remove the radiator from the model, the user can simply set the emissivity to 0 . Secondly, a sublimator is used to release additional heat by converting ice into water vapor. If heat needs to be added to the system, this is done by the third component: a heater. Heat is moved between these three components and the space suit by water-filled tubes. These represent the liquid cooling and ventilation garment (LCVG) currently worn by all astronauts performing EVA. Water from the LCVG is also used to replace the ice lost through sublimation. The astronaut can change the performance of the thermal control system by setting the LCVG temperature at the inlet to the space suit. On the Apollo space suit, this could take three values to give varying levels of cooling: $294 \mathrm{~K}, 288 \mathrm{~K}$, and $280 \mathrm{~K}\left(21^{\circ} \mathrm{C}, 15^{\circ} \mathrm{C}\right.$, and $\left.7^{\circ} \mathrm{C}\right) .^{26}$

The temperature of the LCVG water at the space suit outlet is dependent upon the transfer of heat to or from the space suit, as per the equation:

$$
T_{L C V G, \text { out }}=\frac{\dot{Q}_{\text {total }}}{\dot{m}_{\text {water }} \cdot c_{p, \text { water }}}+T_{L C V G, \text { in }}
$$

There are three conditions under which the thermal control system can operate, based on the total space suit heat flux:

- Heat is being transferred from the space suit to the environment

- Heat is being transferred to the space suit, and the radiator is sufficient to remove all of this heat

- Heat is being transferred to the space suit, but the radiator is not sufficient to remove this heat

In the first case, heat must be added to the space suit. The LCVG water bypasses the radiator and flows through the heater. As stated above, the heater then adds an amount of heat to the water that is equal to the amount removed from the space suit. This brings the temperature of the LCVG water back to the inlet value specified by the astronaut. The flow then bypasses the sublimator and returns to the space suit inlet.

In the second case, the heat being added to the space suit is rejected to deep space by the radiator. This is governed by the same general equation as Eq. (6), with the radiator in place of the entire suit and deep space as the sole environmental component. The radiator is separated from the LCVG water tubes by a gas gap filled with a low pressure gas. ${ }^{48,49}$ This gas facilitates heat transfer between the cooling water and the radiator. However, the gap can 
be evacuated to reduce the heat transfer to the radiator and consequently the temperature of the radiator. This allows the radiator to reject the exact amount of heat that is added to the system, cooling the LCVG water to the correct inlet temperature. The flow then bypasses the sublimator before returning to the space suit.

In the final case, the radiator cannot reject all the heat transferred to the space suit. The sublimator must be used to release all of the remaining heat from the LCVG water by transforming solid water ice into water vapor. This then decreases the water temperature to the space suit inlet value. This method of thermal control has been used in U.S. space suits since the Apollo program. In sublimation, the mass of water required is governed by the equation:

$$
\dot{m}_{\text {sub }}=\frac{\dot{Q}}{h}=\frac{\dot{m}_{\text {water }} c_{p, \text { water }}\left(T_{\text {sub,out }}-T_{\text {sub,in }}\right)}{h}
$$

This brings the temperature of the LCVG water back to the correct inlet temperature. The mass of ice sublimated away is then replenished by the same amount of LCVG water.

\section{B. Rover Power Model}

Power for transportation rovers comes from two different sources: a solar array and batteries. The first can always provide power when in sunlight, but the second is of finite capacity. The limited amount of battery energy can be a concern, as the rover relies on this source of power when there is insufficient sunlight. As a result, the astronauts must ensure that their rover has enough battery power to complete a planned traverse. Unlike the space suit, which cannot replenish its water supply mid-traverse, the rover can recharge its batteries when in the sun through the excess power generated by the solar array.

The power produced by a solar array $\left(P_{S A}\right)$ is described by the following equation:

$$
P_{S A}=V_{s u n} I \eta_{S A} A_{S A}
$$

The solar array efficiency in Eq. (14) is ideal, and does not account for losses due to design inefficiencies, shadowing from the rover itself, or temperature variations. To capture these power losses, SEXTANT multiplies the given efficiency by $0.77 .^{50}$ Equation (14) also assumes that the solar array is vertical and has the ability to rotate to remain perpendicular to the incoming solar rays. This allows for the maximum possible power production. Another assumption is that the solar array is new, and its performance has not degraded with time on the lunar surface. In reality, radiation, thermal outgassing of the material, micrometeroids, and thermal cycling can all decrease the efficiency of a solar array over time. ${ }^{50}$ The user has the ability to change the solar array efficiency in SEXTANT, and can manually decrease the value used in order to account for an older solar array.

The total energy capacity of the batteries is described in part by their specific energy (W-hr/ $\mathrm{kg})$. This is a property of their construction and materials. The specific energy can be multiplied by the total mass of the batteries to give their energy in W-hr. Currently in SEXTANT, the transportation rovers are assumed to have Yardney Technical Products Lithium-Ion (Li-Ion) batteries with a specific energy of $145 \mathrm{~W}-\mathrm{hr} / \mathrm{kg}$. ${ }^{28}$ These were chosen because they have been successfully used for over six years on the Mars Exploration Rovers. The mass of the batteries is assumed to be $269 \mathrm{~kg}$, giving a total energy capacity of $39 \mathrm{~kW}-\mathrm{hr} .{ }^{28}$ While these numbers are currently used within SEXTANT, they are easily modifiable and can be changed by the user to the specifications of any transportation rover.

\section{Rover Energy Consumption During a Traverse}

As with the astronaut thermal model, there are three possible operating conditions:

- The power from the solar array is enough to completely power the rover

- The power from the solar array is not enough to completely power the rover, and the batteries must provide some power

- There is no power from the solar array and the batteries must completely power the rover

In the first case, the batteries are not required at all; the solar array is sufficient. If the solar array is providing more energy than necessary, the excess power can be used to recharge the batteries. If the batteries are already at full capacity, the excess power is shunted out of the rover as heat. This operating condition generally occurs whenever there is complete or nearly-complete sunlight. The second case occurs when there is some sunlight, but not enough to completely power the rover. As a result, the batteries must be called into service to provide the additional power 
required. Finally, in the third case, the rover is completely in shadow and the solar panels do not provide any energy. The batteries are completely responsible for powering the rover.

\section{Example Astronaut and Rover Traverses}

Three example traverses are now presented to demonstrate the capabilities of SEXTANT. The first two are undertaken by suited astronauts, and represent the two possible shadowing conditions. The third traverse is for a transportation rover, and describes how SEXTANT can help to plan a traverse that achieves its scientific goals while remaining within operational constraints. All example traverses undertaken by suited astronauts or transportation rovers in this chapter occur on a $120 \mathrm{~km}$ by $120 \mathrm{~km}$ submap of the area around the lunar south pole, which is the region covered by the LOLA elevation map used in SEXTANT. This is shown in Fig. 8. The south pole is noted by the star, and three prominent craters are labeled. This submap extends $100 \mathrm{~km}$ to the left and above the lunar south pole, and $20 \mathrm{~km}$ to the right and below. Red areas on the 3D mapping interface indicate obstacles as defined by a maximum slope of $15^{\circ}$.

The starting time for each traverse is set to June 4, 2010 at 10:00 am EDT. At this time, the illumination over the entire submap is as presented by Fig. 9 . The blue areas are those in complete shadow $\left(V_{\text {sun }}=0\right)$, and the red areas are in full sunlight $\left(V_{\text {sun }}=1\right)$. The gradient from blue to red (notation 1 on Fig. 9), represents regions of partial shadow $\left(0<V_{\text {sun }}<1\right)$. Figure 9 shows that the left half of the elevation submap is generally in sunlight, while the right half is generally in shadow. It can also be seen that due to the low sun angle, the bottoms of many craters (notations 2 and 3) are in shadow. Similarly, the rim of many craters, like Shackleton (notation 4), rise high enough that they are in sunlight even when the surrounding area is in shadow.

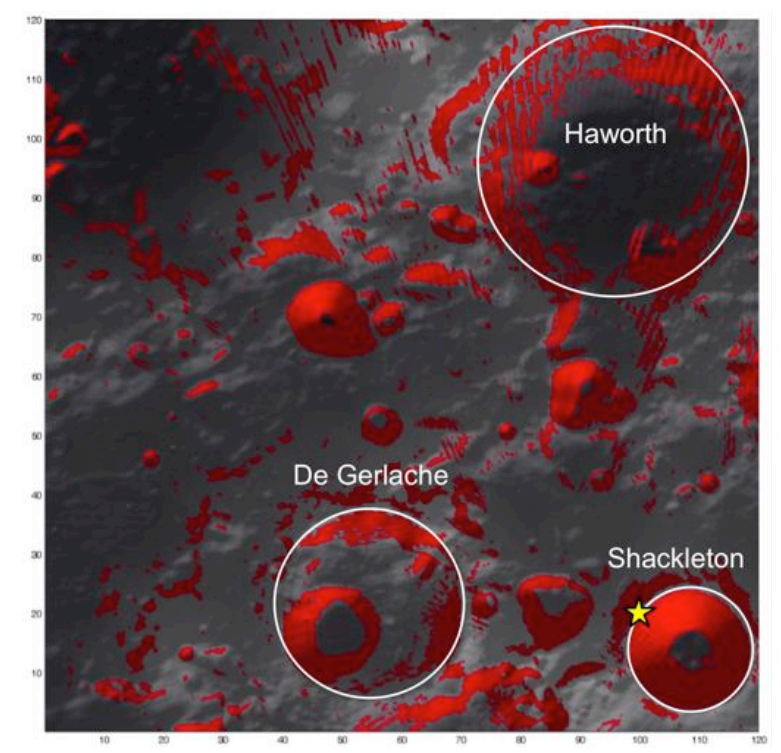

Figure $8.120 \mathrm{~km}$ by $120 \mathrm{~km}$ submap for example traverses.

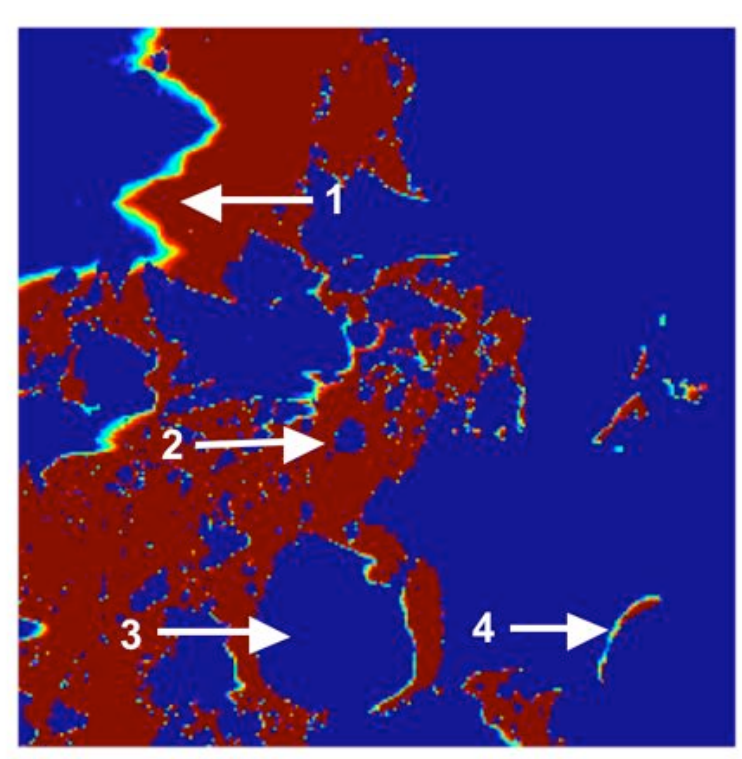

Figure 9. Submap sun illumination on June 4, 2010, at 10:00 am EDT.

\section{A. Astronaut EVA 1 - Traverse in Both Sunlight and Shadow.}

The first example traverse, called EVA 1, travels through areas of both shadow and sunlight. EVA 1 takes place in the lower center of the terrain submap, as shown in Fig. 10. Figure 11 shows a closer view of EVA 1, which is surrounded by the yellow box in Fig. 10. The traverse begins at the habitat located on the rim of de Gerlache Crater (Activity Point 1), and then travels down to the crater's bottom. The traverse does not return to the habitat, and it can be thought that the astronaut rendezvous with a transportation rover at the end. It appears as though the traverse passes through an obstructed area (notation 1 on Fig. 11), but in reality there is a small corridor of traversable ground through with the path travels. The astronaut encounters a $2-\mathrm{km}$ uphill portion right from the start, but then descends $2 \mathrm{~km}$ in altitude over the remainder of the traverse. 




Figure 10. Location of EVA 1 on $120 \mathrm{~km}$ by $120 \mathrm{~km}$ submap.

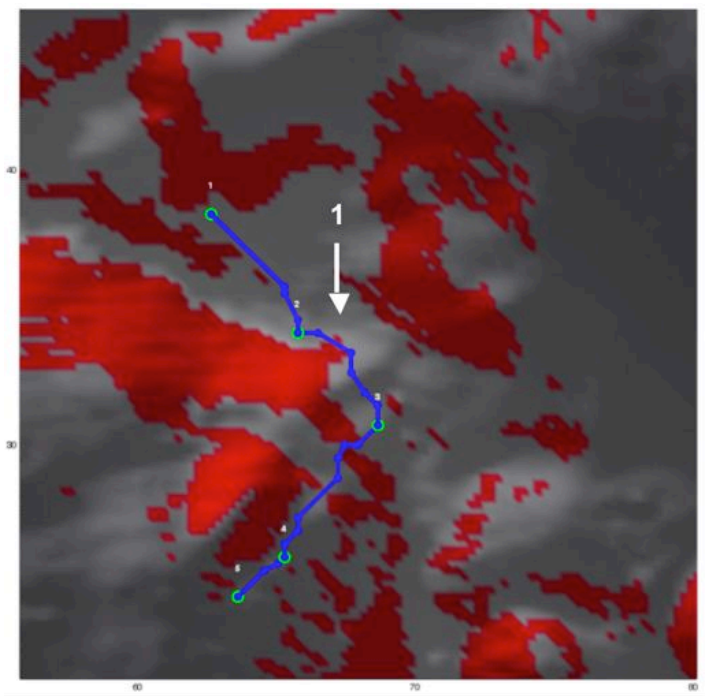

Figure 11. Close-up view of EVA 1 on 20 km by 20 km map.

\section{Traverse Distance, Time, and Metabolic Cost for EVA 1}

Figures 12 and 13 show the metabolic cost of EVA 1 with respect to the traverse distance and time. The red dashed lines represent the constraints of the EVA. These are specified as a distance of $20 \mathrm{~km}$, a time of 8 hours, and a metabolic cost of $12000 \mathrm{~kJ}$. EVA 1 is well within the time and metabolic cost constraints, but much closer to the distance constraint. In Fig. 12, the slope of the blue line is relatively constant, except near the beginning. This implies that the metabolic cost per unit distance does not vary greatly over most of the EVA. Contrarily, the slope of the line in Fig. 13 is not constant for the entire traverse. The portions with a shallower slope (like near the beginning, notation 1) indicate that the metabolic cost per unit time is smaller than the portions of the graph with a steeper slope, like just before Activity Point 2 (notation 2).

\section{Traverse Shadowing and Thermal Metrics for EVA 1}

The traverse shadowing can be seen in Fig. 14. While traveling between the habitat (Activity Point 1) and Activity Point 3, the astronaut is in shadow. Once he crests the rim of de Gerlache just after Activity Point 3, he abruptly transitions into sunlight. The astronaut remains in sunlight until he arrives at the crater floor. Here, there is a more gradual transition into shadow, as seen by the noticeable grey path right before Activity Point 5 (notation 1 on Fig. 14). The astronaut finishes EVA 1 in shadow at Activity Point 5. The shadowing condition at each stage is important because it dictates the thermal equilibrium temperature, which controls the external space suit temperature. This, in turn, influences the amount of heat flux conducted between the environment and the space suit environment through the suit wall. Figure 15 shows the external space suit temperature as a function of the distance and time along EVA 1.

The traverse begins in shadow, where the external space suit temperature is $300 \mathrm{~K}$ and the thermal equilibrium temperature is $102.8 \mathrm{~K}$. Consequently, the external space suit temperature decreases. As the external space suit temperature continues to decrease, more heat is lost from the space suit. The external space suit temperature reaches a minimum of $191.1 \mathrm{~K}$ at 4 hours and 9.5 minutes into the traverse (notation 1 on Fig. 15). The external space suit temperature increases briefly within the partially-shadowed region $\left(V_{\text {sun }}=0.5\right)$ of the traverse mid-way between the Activity Points 2 and 3 (notation 2 on Fig. 15). It then decreases before increasing once again at a much higher rate when the astronaut enters full sunlight (notation 3 on Fig. 15). In both cases, the increase occurs because the thermal equilibrium temperature is above the external space suit temperature. However, the rate is different because of the magnitude of this difference. At the beginning of the partially-shadowed area, the difference between the thermal equilibrium temperature $(237.3 \mathrm{~K})$ to the fourth power and the external space suit temperature $(191.1 \mathrm{~K})$ to the fourth power is $1.04 \cdot 10^{9}$. The same difference at the beginning of the region of complete sunlight is an order of magnitude greater $\left(331.6^{4}-193.4^{4}=1.07 \cdot 10^{10}\right)$. The space suit's temperature reaches a maximum value of $311 \mathrm{~K}$, 
which occurs 6 hours and 47 minutes into the traverse. Whenever the external space suit temperature is greater than $300 \mathrm{~K}$ - the constant internal space suit temperature - the external heat is conduced into the wall of the space suit.

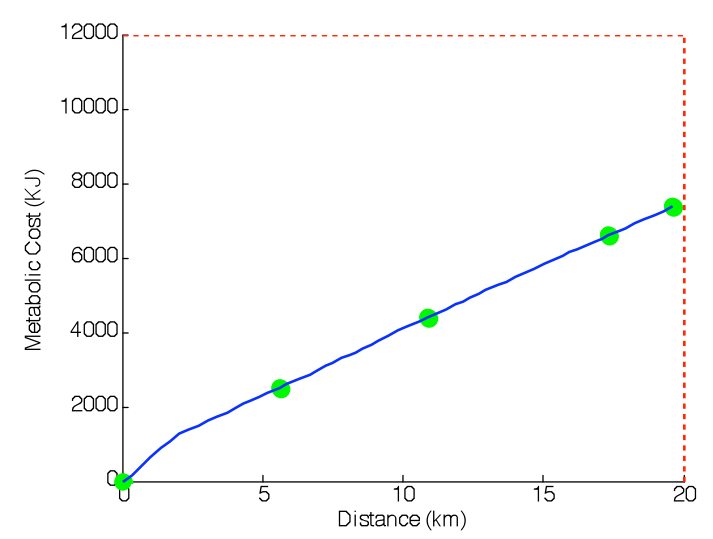

Figure 12. Cumulative metabolic cost of EVA 1 with respect to distance.

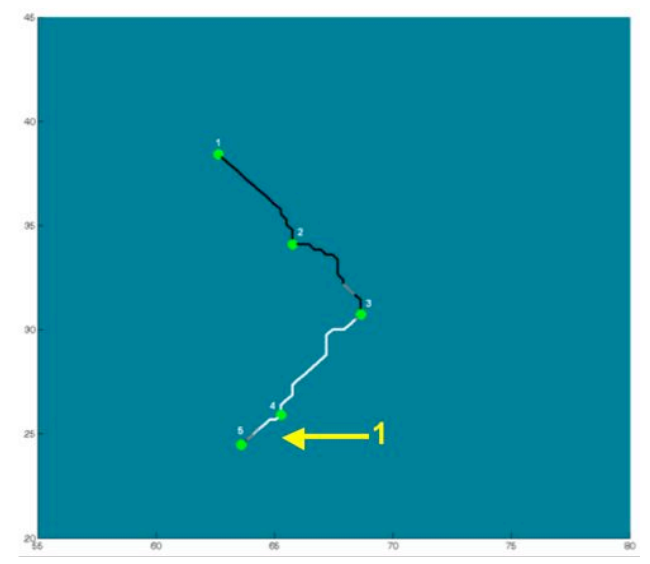

Figure 14. Shadowing along EVA 1.



Figure 16. Mass of sublimated water during EVA 1 with respect to time, with shadowing.

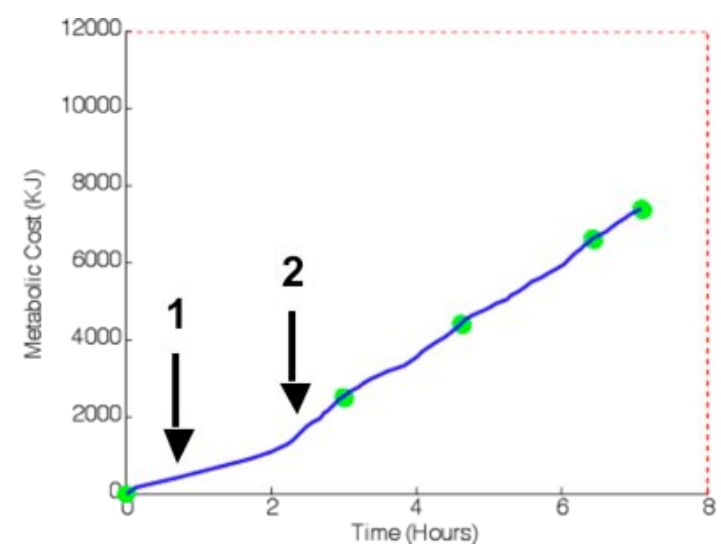

Figure 13. Cumulative metabolic cost of EVA 1 with respect to time.

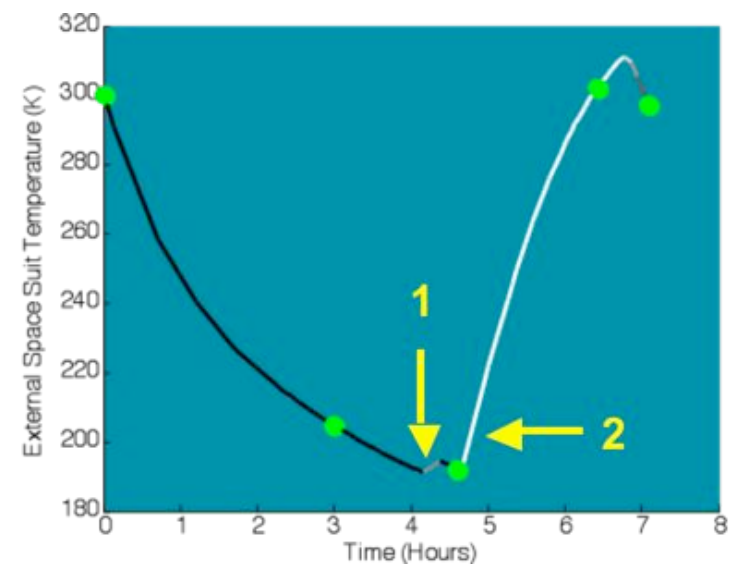

Figure 15. External space suit temperature during EVA 1 with respect to time, with shadowing.

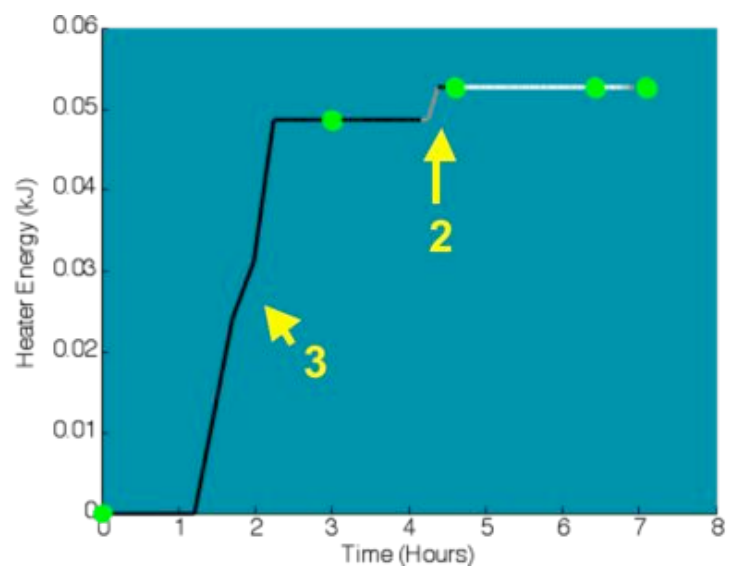

Figure 17. Heater energy during EVA 1 with respect to time, with shadowing. 
Figures 16 and 17 show the mass of water required to replenish ice sublimated away for cooling and the heater energy required for heating. The amount of water required is increasing throughout the traverse; however, the rate at which the water is required varies throughout the traverse. The rate is greatest when the astronaut is in full sunlight near the end of the traverse (notation 1 on Fig. 16). It is slower elsewhere, and even decreases to $0 \mathrm{~kg} / \mathrm{hr}$ at two periods (notations 2 and 3 on Fig. 16). During these periods, no cooling is being used by the space suit. Rather, from looking at Fig. 17, it can be seen that heating is required during these periods (notations 2 and 3). This is a function of the design of the thermal control system - either cooling or heating are required, never both at the same time. For the entire traverse, approximately $2.5 \mathrm{~kg}$ of water are consumed. The Apollo space suits had $5 \mathrm{~kg}$ of water for cooling purposes, ${ }^{51}$ twice as much as this traverse requires. Furthermore, the amount of heater power required is approximately $50 \mathrm{~J}-\mathrm{a}$ trivial amount. These metrics show that EVA 1 is well within the space suit's thermal constraints, and is considered feasible.

\section{B. Astronaut EVA 2 - Traverse Entirely in Shadow}

The second example astronaut traverse, EVA 2, represents the opposite extreme case of the astronaut thermal loading - when the entire traverse is in complete shadow. Figure 18 shows the traverse on the $120 \mathrm{~km}$ by $120 \mathrm{~km}$ submap, and Fig. 19 shows a close-up of the path. The elevation profile of the traverse is roughly symmetric, where the astronaut travels uphill to Activity Point 3 and then continues downhill. The maximum positive slope is $11.4^{\circ}$, and the maximum negative slope is $-14.3^{\circ}$.



Figure 18. Location of EVA 2 on $120 \mathrm{~km}$ by $120 \mathrm{~km}$ submap.

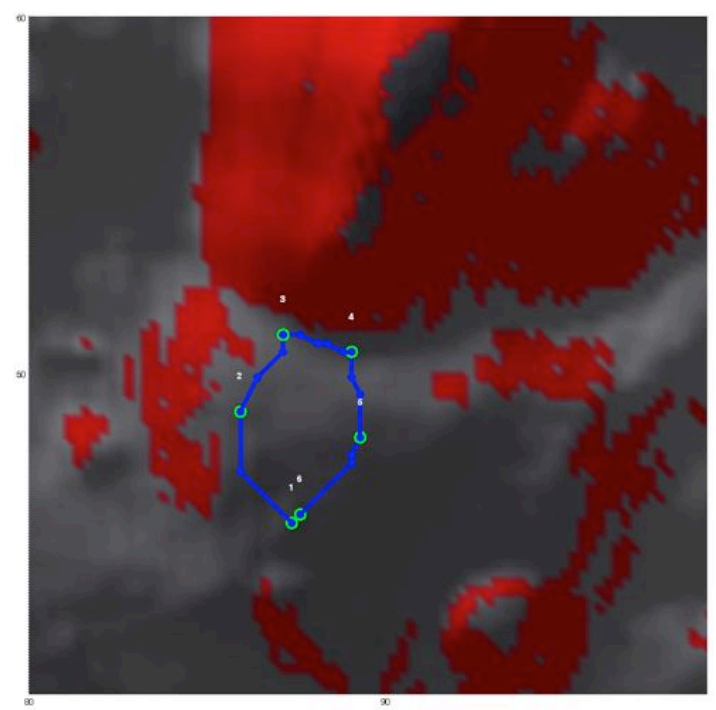

Figure 19. Close-up view of EVA 2 on $20 \mathrm{~km}$ by $20 \mathrm{~km}$ map.

\section{Traverse Distance, Time, and Metabolic Cost for EVA 2}

During the EVA, the astronaut stops at some of the Activity Points and performs scientific activities. He stops for 30 minutes at Activity Points 1, 4, and 5; 15 minutes at Activity Point 2; and 45 minutes at Activity Point 3. When an astronaut remains stationary at any Activity Point, he is assumed to have a metabolic rate of $280 \mathrm{~W}$ - the average metabolic rate of the Apollo astronauts performing scientific activities on the lunar surface. ${ }^{26}$ Figures 20 and 21 show the metabolic cost of EVA 2 with respect to distance and time. The green regions represent when the astronaut is working at the Activity Points. As was stated above, the metabolic cost of a traverse increases as the astronaut works at an Activity Point. However, the traverse distance does not change, as the astronaut is considered to be "stationary" for that time period (although he is certainly working and moving around locally). This can be seen in Fig. 20, as the blue line is completely vertical at the Activity Points. On the other hand, the traverse time does increase while the astronaut is working at an Activity Point. Because the metabolic rate is a constant value for all Activity Points, the slope of the blue line in Fig. 21 is constant at all Activity Points. 


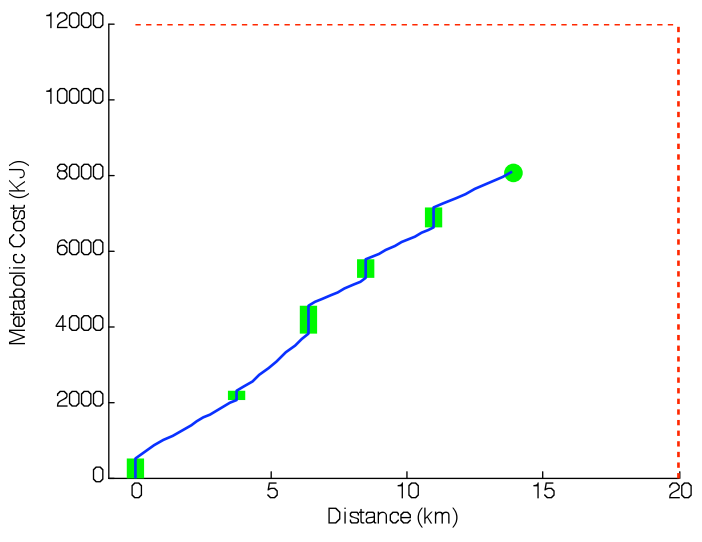

Figure 20. Cumulative metabolic cost of EVA 2 with respect to distance.

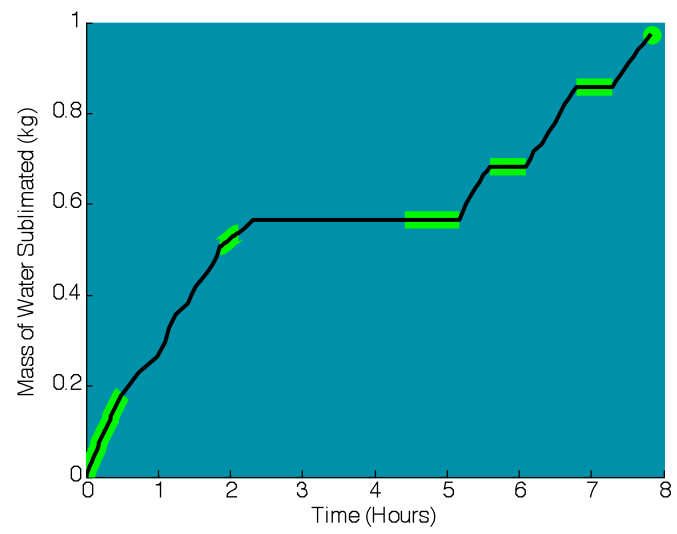

Figure 22. Mass of sublimated water during EVA 2 with respect to time, with shadowing.

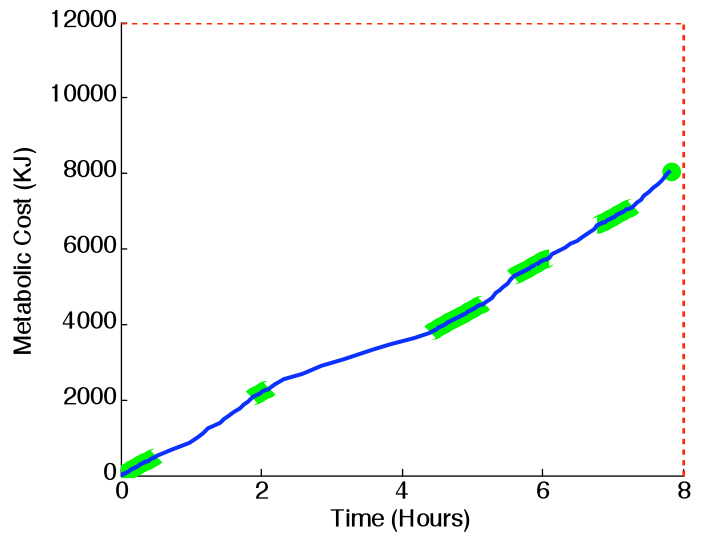

Figure 21. Cumulative metabolic cost of EVA 2 with respect to time.

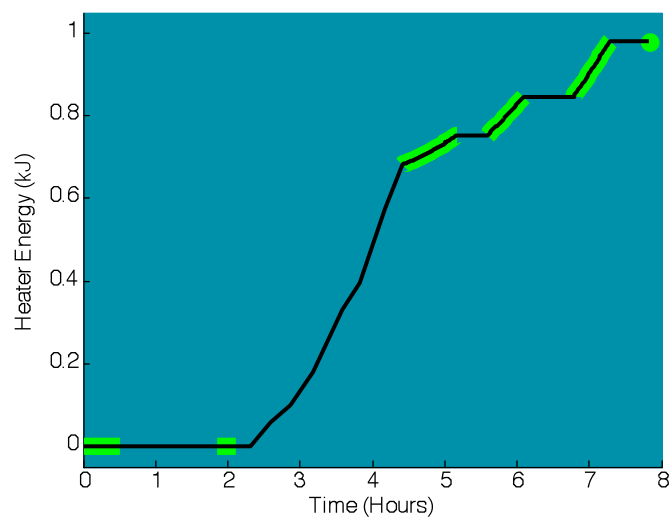

Figure 23. Heater energy during EVA 2 with respect to time, with shadowing.

\section{Traverse Shadowing and Thermal Metrics for EVA 2}

The traverse shadowing shows that the astronaut is indeed in complete shadow during the entire traverse. Figures 22 and 23 show the amount of water required for sublimation and heater power used during EVA 2. Contrary to what one might think, EVA 2 - and any traverse in complete shadow - does not require only heating. Sublimation cooling is still necessary during some stages of the traverse. In the beginning of the traverse, the external space suit temperature is very close to the internal space suit temperature of $300 \mathrm{~K}$, and so there is minimal heat loss to the environment. As a result, the electronics waste heat flux and astronaut metabolic heat flux are enough to overwhelm this loss, and the net heat transfer is still into the space suit. Cooling is therefore required.

As the traverse continues, the external space suit temperature smoothly decreases to the thermal equilibrium temperature of $102.8 \mathrm{~K}$ and the heat loss to the environment increases. Eventually, the total heat flux becomes negative, and heat is lost from the space suit to the environment. This occurs because the astronaut is traveling across steep terrain and moving slowly, and is not producing a lot of excess metabolic heat. This source of heat, combined with the electronics waste heat, is not enough to surpass the heat loss to the environment.

During subsequent movements, the astronaut is traveling downhill. As a result, the metabolic heat flux and the electronics waste heat combined are greater than the external heat flux, even though the external space suit temperature is low. The net heat transfer is to the space suit, and sublimation cooling is used to remove this excess heat. Whenever the astronaut is working at an Activity Point, he is not using as much energy as while walking. The conduction heat transfer through the space suit to the environment dominates the electronics waste heat flux and the metabolic heat flux, and heating is once again required. 


\section{Rover Traverse}



Figure 24. Location of rover traverse on submap.

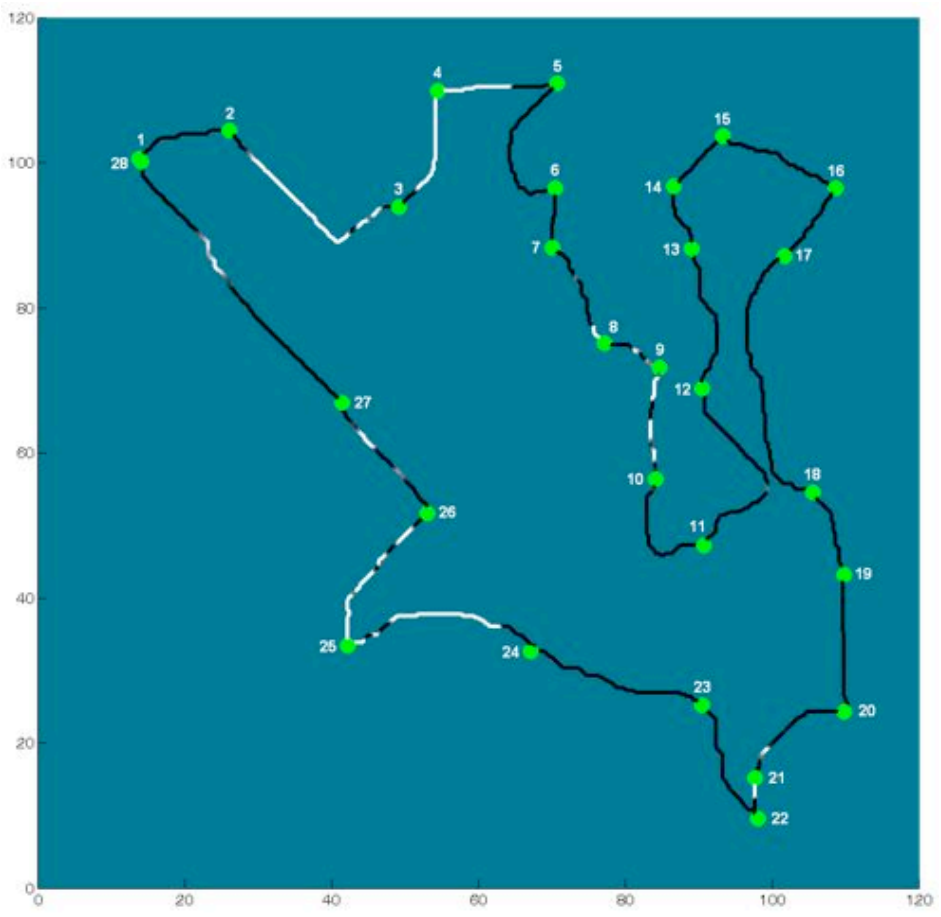

Figure 25. Shadowing along rover traverse.

\section{Planning the Desired Traverse}

For traverses with a transportation rover, SEXTANT focuses on how the shadowing affects the rover's power system. The main metric calculated is the energy level of the rover's batteries. Because explorations of the lunar poles will encounter large shadowed areas, it is important for astronauts to ensure that the rover's batteries have enough energy to complete a given traverse and return home. The batteries can be recharged by the solar array if it is producing excess power. One example traverse is presented here that shows how a rover traverse can be planned to accomplish its scientific goals while fitting all power constraints.

As shown in Fig. 24, the traverse originates from the habitat (Activity Point 1), in a low point in the upper-left corner of the map. The rover begins by remaining stationary for 60 minutes, then climbing a hill to gain $5.8 \mathrm{~km}$ of altitude between the habitat and Activity Point 5. Once the rover crests the hill, it travels downhill around the rim of Haworth Crater. The rover stops at Activity Points 6 and 7 for 30 minutes and 60 minutes, respectively, for scientific observations. The rover then continues around the crater rim, stopping at Activity Point 10 for 60 minutes to examine the small, deep crater just south of Haworth. The rover then travels down into Haworth Crater to explore its floor. Besides the habitat, this is the lowest point reached on the traverse. The rover stops for a total of 6 hours in this region, distributed amongst Activity Points $13-17$. The longest single stop is for 180 minutes at Activity Point 16. Exploring the bottom of craters is an important task, as these areas expose lower portions of the Moon's crust that are very scientifically interesting. The bottom of Haworth Crater is also a permanentlyshadowed region, where there is the greatest chance of finding water ice or other volatiles. ${ }^{43}$ Once the exploration of the Haworth Crater floor is complete, the rover travels out of the crater and to the rim of Shackleton Crater. The rover spends 30 minutes at Activity Point 18, and 90 minutes at both Activity Points 20 and 22 before traveling back towards the habitat. Along the way, the rover stops at Activity Points $24-27$ for varying amounts of time between 15 and 60 minutes, in order to explore additional craters. In total, this rover traverse 
covers $533.50 \mathrm{~km}$, lasts 50 hours and 17 minutes (with stops), and uses $331.8 \mathrm{GJ}$ of energy.

Figure 25 shows the shadowing along the rover traverse. The traverse begins in shadow, but reaches an area of sunlight between Activity Points 2 and 5. After Activity Point 5, the rover spends most of its time in shadow as it circles Haworth Crater and then descends to the crater floor. There are brief periods of sunlight around Activity Points 9 and 10. More sunlight is encountered while on the rim of Shackleton Crater, around Activity Point 21. The portion of the traverse from Shackleton Crater back to the habitat has some sunlit portions, mainly between Activity Points 24 and 26. All in all, the rover spends $78.3 \%$ of its time in complete shadow, $8.2 \%$ in partial shadow, and $13.5 \%$ in complete sunlight. Because the rover spends so much time in complete shadow, this traverse will require a great deal of battery power. It will be essential to make sure that the rover battery is large enough so that it never runs out of energy. The rover's battery energy level is plotted as a function of both time and distance along the traverse in Fig. 26.

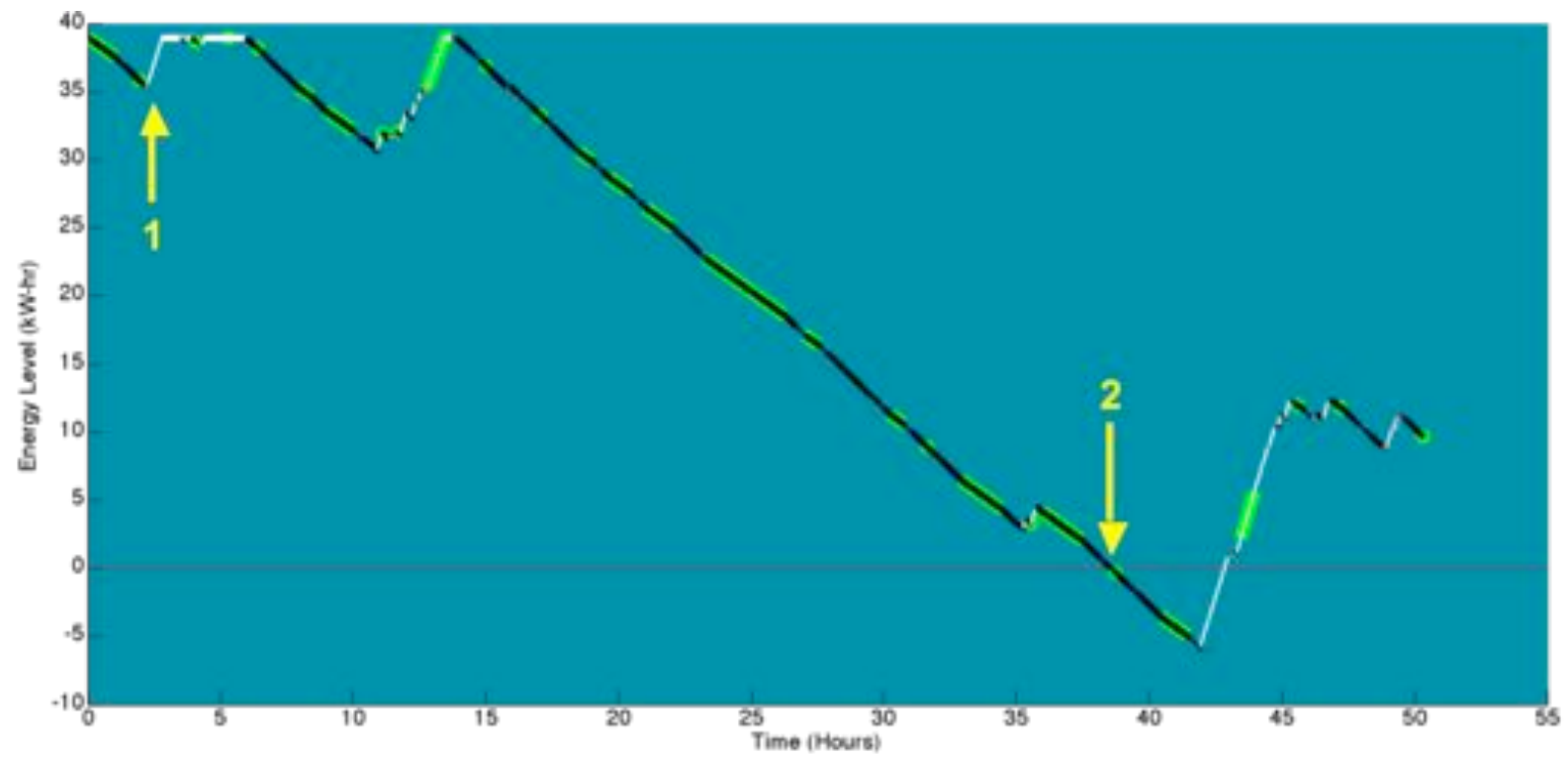

Figure 26. Battery energy level during original rover traverse.

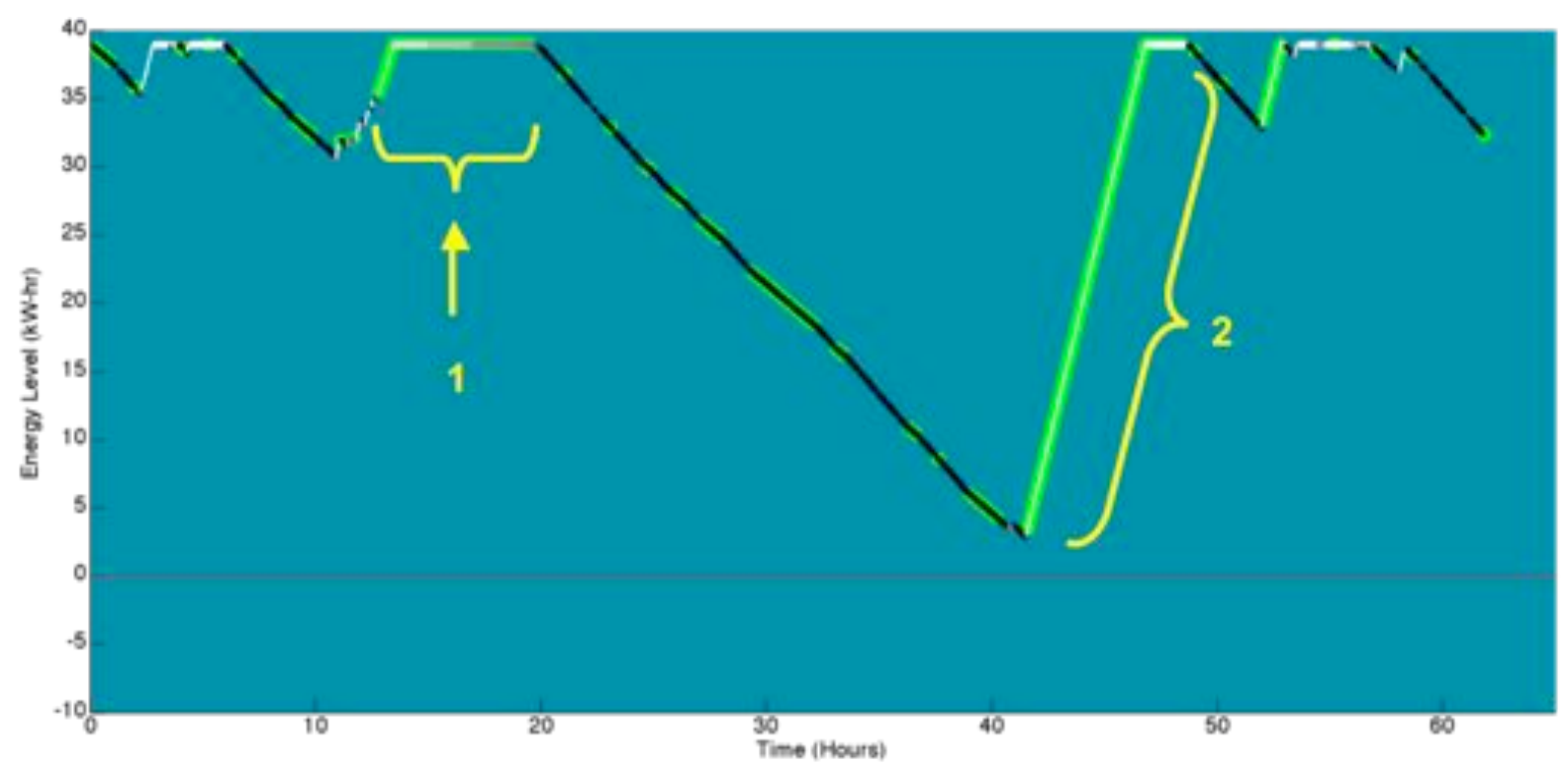

Figure 27. Battery energy level during replanned traverse. 
The traverse begins in darkness, and so the solar array cannot produce any power. The rover must rely on batteries. The batteries discharge from their initial level of $39 \mathrm{~kW}-\mathrm{hr}$ to $35.4 \mathrm{~kW}-\mathrm{hr}$ in 2 hours and 11 minutes. At this point, the rover enters the first sunlit region (notation 1 on Fig. 26). The solar array is sized such that it produces more than enough power to run the rover when in complete sunlight. The excess power is used to recharge the battery. Once the battery regains its maximum energy level, the excess power produced by the solar arrays is not needed, and is shunted off as heat. This discharge-recharge cycle continues, as the rover uses battery energy between Activity Points 5 and 9, and recharges once again to the full level. However, after Activity Point 10, the rover spends a long period of time in complete shadow, powering itself solely by the batteries. There is no opportunity for recharging. This leads to a major problem, as the rover's energy level reaches $0 \mathrm{~W}$ right before Activity Point 23, 38 hours and 34 minutes after the traverse begins (notation 2). This would be a catastrophic failure, as the rover is over $100 \mathrm{~km}$ away from the habitat, an unrealistic distance for astronauts to travel on foot. So, modifications must be made to the traverse to spend more time in sunlight, either while moving or stationary. These modifications can be accomplished in conjunction with another task - to add long-duration stops along the traverse where the astronauts can sleep. It is important to ensure that these stops occur in the sunlight so that the solar arrays can completely power the rover, and that the batteries can recharge if depleted.

Two long-duration stops have been added to the traverse to allow the astronauts to sleep. Firstly, the stop at Activity Point 10 has been extended from 60 minutes to 7 hours. Secondly, the 90 minute stop at Activity Point 22 has been cancelled, and a 7 hour stop at Activity Point 21 has been added. These two sleep periods split the traverse into three waking "days" of 12 hours and 44 minutes, 21 hours and 50 minutes, and 13 hours and 13 minutes. While the second day is a bit longer than desired, Activity Point 21 is the best place to stop as it is located within the only sunlit area in the middle of the traverse. While stopped at these Activity Points the rover battery has an opportunity to completely recharge (notations 1 and 2 on Fig. 27), which prevents the rover from running out of power along the traverse. The rover battery energy level with these stops is shown in Fig. 27. The minimum energy level reached is $2.87 \mathrm{~kW}-\mathrm{hr}$, a margin of $7.3 \%$. So, the traverse is deemed to be feasible now that a sufficient number of sleep periods have been added and the energy constraints have been satisfied.

\section{Generating Return-Home Paths}

One final important consideration is the amount of energy that it takes to return directly to the habitat from points along the traverse. It may be the case that the most energy-efficient path computed by SEXTANT from a certain point back to the habitat takes the rover through shadowed areas where the battery energy is completely exhausted. If this is the case, there are two options: the designated return-home path from this point can be one that is not the most energy-efficient route, but takes the rover through more sunlight; or the traverse itself can be modified so that the rover always has enough energy to return to the habitat. SEXTANT allows the user to specify return-home paths from any point along the traverse and to determine the shadowing and battery energy level along this route. This

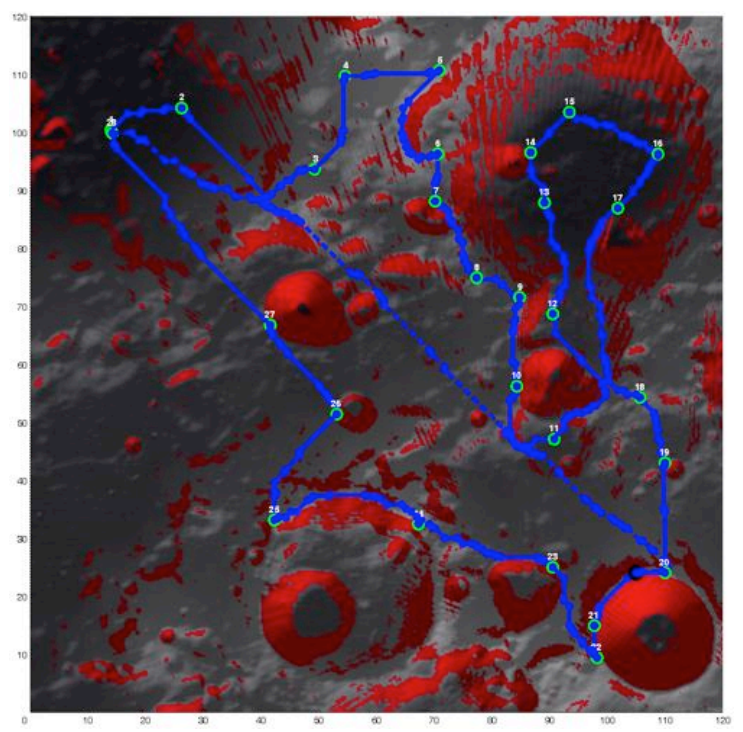

Figure 28. Rover return-home path from Shackleton Crater to habitat.



Figure 29. Shadowing along rover return-home path.

25

American Institute of Aeronautics and Astronautics 
allows him to test whether or not the energy constraints would be violated on a return-home path. As stated before, the rover energy level at the beginning of the return-home path is the value that it had at that particular point in the parent traverse.

This example return-home path begins between Activity Points 20 and 21, on the rim of Shackleton Crater. It is $135.44 \mathrm{~km}$ back to the habitat, which takes 9 hours and 1 minute and $64.3 \mathrm{GJ}$ of energy. This route begins in shadow and then eventually enters portions of sunlight (Fig. 29). During this return-home path, the rover battery is completely discharged after only 2 hours. This is because the initial energy level is low - only $3.574 \mathrm{~kW}-\mathrm{hr}$. In an emergency, the rover would not be able to complete this path back home. If the original traverse was not modified, in an emergency the rover would have to travel to Activity Point 21, remain stationary while the solar arrays recharged the batteries enough, and then continue on directly to the habitat. This would take additional time, and is certainly undesirable. The user is able to modify the original traverse, in order to a solution that satisfies the energy constraints for both the original traverse and the return-home paths from points along the traverse. In the future, SEXTANT can be made to automatically check the return-home paths from points along the traverse, to see if the rover has enough energy to return home. If so, the user can be alerted and can make a decision on how best to modify the traverse.

\section{Conclusion}

Even though the specific goals of lunar and planetary exploration are unknown, NASA will undoubtedly reach out to the Moon and Mars with both manned and robotic missions. While the timeline for these missions is not yet firm, it is still crucial to begin developing the next generation of technology that will assist these explorers, like SEXTANT. As this paper has shown, SEXTANT contains the most comprehensive and accurate representation of lunar traverses of any tool. It can be used to plan safe, efficient, and effective traverses across the lunar surface for suited astronauts and transportation rovers. By computing the shadowing along traverses, SEXTANT can determine the thermal consumables of astronauts and the power usage and generation of transportation rovers. These metrics allow an explorer to check if his traverse is feasible with high fidelity well before he leaves the habitat. As the first step towards a tool giving explorers the autonomy to plan their own paths, SEXTANT has been integrated with iMAS. This capability will allow for more efficient and ambitious traverse planning, as less communication with Earth will be required. The capabilities of SEXTANT can also be used to realistically simulate traverses on the Moon and Mars years before humans actually set foot on the surface. This is beneficial to hardware designers, as SEXTANT's ability to model the shadowing-related consumables of sample traverses can help to properly size the thermal control system of space suits and the electrical power systems of transportation rovers required for future explorations. With its current functionality and future improvement, SEXTANT will be an invaluable mission planning tool for future lunar and Martian explorers.

\section{Acknowledgments}

The authors wish to thank Dr. Jessica Márquez, John Dowding, Ron van Hoof, Dr. Maarten Sierhuis, and Dr. William Clancey from NASA Ames Research Center for their assistance in integrating SEXTANT and iMAS. Funding for this research was provided by the MIT Donald W. Douglas Fellowship and the National Space Biomedical Research Institute Grant HFP00003.

\section{References}

${ }^{1}$ Culbert, C., "Lunar Surface Systems Project Overview", U.S. Chamber of Commerce, Feb. 2009.

${ }^{2}$ Carr, C.E., Newman, D.J., and Hodges, K., "Geologic Traverse Planning for Planetary EVA”, SAE, Paper 2003-01-2416, July 2003.

${ }^{3}$ Jones, E.M., "Climbing Cone Ridge - Where Are We?", Apollo Lunar Surface Journal [online database], URL: http://www.hq.nasa.gov/alsj/a14/a14.tocone.html [cited 3 Aug. 2010].

${ }^{4}$ Parasuraman, R., Sheridan, T.B., and Wickens, C.D., "A Model for Types and Levels of Human Interaction with Automation", IEEE Transactions on Systems, Man, and Cybernetics - Part A: Systems and Humans, Vol. 30, No. 3, 2000, pp. 286-297.

${ }^{5}$ Parasuraman, R., Riley, V., "Humans and Automation: Use, Misuse, Disuse, Abuse”, Human Factors, Vol. 39, No. 2, 1997, pp. 230-253.

${ }^{6}$ Layton, C., Smith, P.J., McCoy, C.E., "Design of a Cooperative Problem-Solving System for En-Route Flight Planning: An Empirical Evaluation”, Human Factors, Vol. 36, No. 1, 1994, pp. 94-119.

${ }^{7}$ Smith, P.J., McCoy, C.E., Layton, C., "Brittleness in the Design of Cooperative Problem-Solving Systems: The Effects on User Performance", IEEE Transactions on Systems, Man, and Cybernetics - Part A: Systems and Humans, Vol. 27, No. 3, 1997, pp. 360-371. 
${ }^{8}$ Norris, J.S., Powell, M.W., Vona, M.V., Backes, P.G., Wick, J.V., "Mars Exploration Rover Operations with the Science Activity Planner", Proceedings of the 2005 IEEE International Conference on Robotics and Automation, IEEE, New York, 2005, pp. 4629-4634. 2009.

${ }^{9}$ McCurdy, M., "Planning Tools for Mars Surface Operations: Human-Computer Interaction Lessons Learned", IEEE, March

${ }^{10}$ Muehlberger, W.R., “Apollo 16 Traverse Planning and Field Procedures”, Geological Survey Professional Paper 1048: Geology of the Apollo 16 Aarea, Central Lunar Highlands, edited by G.E. Ulrich, C.A. Hodges, and W.R. Muehlberger, U.S. Government Printing Office, Washington, DC, 1981, pp. 10-20.

${ }^{11}$ Wilkinson, N., "Implementation of a Traverse Generation Assistant for Long-Distance Pressurized Rover Expeditions", Martian Expedition Planning, edited by C. S. Cockell, Science and Technology Series, Vol. 107, American Astronautical Society, San Diego, CA, 2004, pp 259-272.

${ }^{12}$ Wood, B.M., and Wood, Z.J., "Energetically Optimal Travel across Terrain: Visualizations and a New Metric of Geographic Distance with Archaeological Applications”, SPIE, Paper 6060-15, Jan. 2006.

${ }^{13}$ Santee, W.R., Allison, W.F., Blanchard, L.A., and Small, M.G., “A proposed model for load carriage on sloped terrain”, Aviation, Space, and Environmental Medicine, Vol. 72, No. 6, 2001.

${ }^{14}$ Carr, C.E., Distributed Architectures for Mars Surface Exploration, M.S. Thesis, Dept. of Aeronautics and Astronautics, Massachusetts Institute of Technology, Cambridge, MA, 2001.

${ }^{15}$ Márquez, J.J., Human-Automation Collaboration: Decision Support for Lunar and Planetary Exploration, Ph.D. Dissertation, Dept. of Aeronautics and Astronautics, Massachusetts Institute of Technology, Cambridge, MA, 2007.

${ }^{16}$ Márquez, J.J., and Cummings, M.L., "Design and Evaluation of Path Planning Decision Support for Planetary Surface Exploration", Journal of Aerospace Computing, Information, and Communication, Vol. 5, No. 5, 2008, pp. 57-71.

${ }^{17}$ Márquez, J.J., Cummings, M.L., Roy, N., Kunda, M., and Newman, D.J., "Collaborative Human-Computer Decision Support for Planetary Surface Traversal”, AIAA, Paper 2005-6993, Sept. 2005.

${ }^{18}$ Márquez, J.J., and Newman, D.J., "Planning and Re-planning for Planetary Extravehicular Activities: Analysis of Excursions in a Mars-Analog Environment and Apollo Program", SAE, Paper 2006-01-2297, July 2006.

${ }^{19}$ Márquez, J.J., and Newman, D.J., "Recommendations for Real-Time Decision Support Systems for Lunar and Planetary EVAs", SAE, Paper 2007-01-3089, July 2007.

${ }^{20}$ Lindqvist, L.V.J., Multidisciplinary Extravehicular Activity Mission Optimization for Lunar Exploration, M.S. Thesis, Institute of Astronautics, Technishe Universität München, Munich, Germany, 2008.

${ }^{21}$ Essenburg, J.R., Mission Planning and Navigation Support for Lunar and Planetary Exploration, M.S. Thesis, Dept. of Aeronautics and Astronautics, Massachusetts Institute of Technology, Cambridge, MA, 2008.

${ }^{22}$ Smith, D.E., Zuber, M.T., Torrence, M.H., McGarry, J.F., Pearlman, M., "Laser Ranging to the Lunar Reconnaissance Orbiter (LRO)", International Laser Ranging Service, Oct. 2006.

${ }^{23}$ Ramos-Izquierdo, L., Scott III, V.S., Connelly, J., Schmidt, S., Mamakos, W., Guzek, J., Peters, C., Liiva, P., Rodriguez, M., Cavanaugh, J., Riris, H., "Optical System Design and Integration of the Lunar Orbiter Laser Altimeter", Applied Optics, Vol. 48, No. 16, 2008, pp. 3035-3049.

${ }^{24}$ Smith, D.E., Zuber, M.T., Jackson, G.B., Cavanaugh, J.F., Neumann, G.A., Riras, H., Sun, X., Zellar, R.S., Coltharp, C., Connelly, J., Katz, R.B., Kleyner, I., Liiva, P., Matuszeski, A., Mazarico, E.W., McGarry, J.F., Novo-Gradac, A., Ott, M.N., Peters, C., Ramos-Izquierdo, L.A., Ramsey, L., Rowlands, D.D., Schmidt, S., Scott III, V.S., Shaw, G.B., Smith, J.C., Swinski, J., Torrence, M.H., Unger, G., Yu, A.W., Zagwodzki, T.W., "The Lunar Orbiter Laser Altimeter Investigation on the Lunar Reconnaissance Orbiter Mission”, Space Science Review, Vol. 150, No. 1-4, 2010, pp. 209-241.

${ }^{25}$ Smith, D.E., Zuber, M.T., Neumann, G.A., Lemoine, F.G., Mazarico, E., Torrence, M.H., McGarry, J.F., Rowlands, D.D., Head, J.W., Duxbury, T.H., Aharonson, O., Lucey, P.G., Robinson, M.S., Barnouin, O.S., Cavanaugh, J.F., Sun, X., Liiva, P., Mao, D., Smith, J.C., Bartels, A.E., "Initial Observations from the Lunar Orbiter Laser Altimeter (LOLA)", Geophysical Research Letters (to be published).

${ }^{26}$ Waligora, J.M., and Horrigan, D.J., "Metabolism and Heat Dissipation during Apollo EVA Periods", Biomedical Results of Apollo, edited by R.S. Johnston, L.F. Dietlein, and C.A. Berry, NASA SP-368, 1975, pp. 115-128.

${ }^{27}$ Heiken, G.H., Vaniman, D.T., and French, B.M., Lunar Sourcebook, Cambridge University Press, New York, 1991.

${ }^{28}$ Hong, S., Design of Power Systems for Extensible Surface Mobility Systems on the Moon and Mars, M.S. Thesis, Dept. of Aeronautics and Astronautics, Massachusetts Institute of Technology, Cambridge, MA, 2007.

${ }^{29}$ Hart, P.E., Nilsson, N.J., and Raphael, B., “A Formal Basis for the Heuristic Determination of Minimum Cost Paths”, IEEE Transactions on Systems, Science, and Cybernetics, Vol. 4, No. 2, 1968, pp. 100-107.

${ }^{30}$ Johnson, A.W., An Integrated Traverse Planner and Analysis Tool for Future Lunar Surface Explorations, M.S. Thesis, Dept. of Aeronautics and Astronautics, Massachusetts Institute of Technology, Cambridge, MA, 2010.

${ }^{31}$ Rupert S., and Boston, P., "Science Reports", Mars Desert Research Station Crew 41 Log Book [online database], URL: http://desert.marssociety.org/MDRS/fs05/0109/sci.asp [cited 4 Aug. 2010].

${ }^{32}$ Garry, B., "EVA Reports", Mars Desert Research Station Crew 46 Log Book [online database], URL: http://desert.marssociety.org/MDRS/fs05/0503/eva.asp [cited 4 Aug. 2010].

${ }^{33}$ Bleacher, J., Garry, B., Zimbelman, J., Clancey, W., Sierhuis, M., "The use of individual Mobile Agents Systems (iMAS) in the field: A geologist's perspective on value for future planetary missions”, NASA Lunar Science Institute, Poster, July 2009. 
${ }^{34}$ Clancey, W.J., Sierhuis, M., Alena, R.L., Berrios, D., Dowding, J., Graham, J.S., Hirsh, R.L., Garry, W.B., Semple, A., Rupert, S.M., and van Hoof, R.J.J., "Planning for Exploration: The Mobile Agents 2005 Field Test at MDRS", The Mars Society, Aug. 2005.

${ }^{35}$ Clancey, W.J., Sierhuis, M., Alena, R.L., Berrios, D., Dowding, J., Graham, J.S., Tyree, K.S., Hirsh, R.L., Garry, W.B., Semple, A., Buckingham Shum, S.J., Shadbolt, N. and Rupert, S., Automating CapCom using Mobile Agents and Robotic Assistants, NASA 2007-214554, 2007.

${ }^{36}$ Clancey, W.J, Sierhuis, M., Alena, R.L., Crawford, S., Dowding, J., Graham, J., Kaskiris, C., Tyree, K.S., and van Hoof, R.J.J., "The Mobile Agents integrated field test: The Mars Desert Research Station April 2003", Proceedings of the 17 ${ }^{\text {th }}$ International FLAIRS Conference, Florida AI Research Society, May 2004, pp. 732-737.

${ }^{37}$ Clancey, W.J., Sierhuis, M., Alena, R.L., Dowding, J., Scott, M., van Hoof, R., "Power System Agents: The Mobile Agents 2006 Field Test at MDRS", The Mars Society, Aug. 2006.

${ }^{38}$ Clancey, W.J., Sierhuis, M., Kaskiris, C. and van Hoof, R.J.J., "Advantages of Brahms for specifying and implementing a multiagent human-robotic exploration system", Proceedings of The 16th International FLAIRS Conference, Florida AI Research Society, May 2003, pp. 7-11.

${ }^{39}$ Sierhuis, M., Clancey, W.J., and van Hoof, R.J.J., "Brahms: a multi-agent modeling environment for simulating work processes and practices", International Journal of Simulation and Process Modelling, Vol. 3, No. 3, 2007, pp. 134-152.

${ }^{40}$ Kuznetz, L.H., "Designing a Smart Suit for the Moon and Mars", SAE, Paper 2008-01-1952, July 2008.

${ }^{41}$ Garrick-Bethell I., Byrne, S., Hoffman, J.A., and Zuber, M.T., "Areas of Favorable Illumination at the Lunar Poles Calculated from Topography", Lunar and Planetary Institute, Abstract 2006, March 2005.

${ }^{42}$ Mazarico, E., Neumann, G.A., Smith, D.E., Zuber, M.T., "Illumination Conditions in the Lunar Polar regions from Lunar Orbiter Laser Altimeter (LOLA) Data", American Geophysical Union, Poster U31A-0007, Dec. 2009.

${ }^{43}$ Mazarico, E., Neumann, G.A., Smith, D.E., Zuber, M.t., Torrence, M.H., "Illumination Conditions of the Lunar Polar Regions Using LOLA Topography”, Icarus (submitted for publication).

${ }^{44}$ Carr, C.E., and Newman, D.J., "Space Suit Bioenergetics: Framework and Analysis of Unsuited and Suited Activity", Aviation, Space, and Environmental Medicine, Vol. 78, No. 11, 2007, pp. 1013-1022.

${ }^{45}$ Fiala, D., Lomas K. J., Stohrer M., "A computer model of human thermoregulation for a wide range of environmental conditions: the passive system”, Journal of Appiled Physiology, Vol. 87, No. 5, 1999, pp. 1957-1972.

${ }^{46}$ Margaria, R., Biomechanics and Energetics of Muscular Exercise, Oxford University Press, Oxford, United Kingdom, 1976.

${ }^{47}$ Mosteller, R.D., "Simplified Calculation of Body Surface Area”, New England Journal of Medicine, Vol. 317, No. 17, 1987.

${ }^{48}$ Campbell, A.B., French, J.D., Nair, S.S., Miles, J.B., Lin, C.H., "Thermal Analysis and Design of an Advanced Space Suit", Journal of Thermophysics and Heat Transfer, Vol. 14, No. 2, 2000, pp. 151-160.

${ }^{49}$ Trevino, L., Copeland, R.J., Elliott, J.E., Weislogel, M., "Freeze Tolerant Radiator for Advanced EMU”, SAE, Paper 200401-2263, July 2004.

${ }^{50}$ Wertz, J.R., and Larson, W.J., Space Mission Analysis and Design, 3rd ed., Springer, New York, 1999.

51“Apollo 17 Traverse Plannning Procedures", Apollo Lunar Surface Journal [online database], URL: http://history.nasa.gov/alsj/a17/A17TraversePlanningData.pdf [cited 8 Aug. 2010]. 